\title{
Magnetohydrodamic and kinetic scale turbulence in the near-Earth space plasmas: A (short) biased review
}

\author{
Fouad Sahraoui · Lina Hadid · Shiyong Huang
}

Received: date / Accepted: date

\begin{abstract}
The near-Earth space is a unique laboratory to explore turbulence and energy dissipation processes in magnetized plasmas thanks to the availability of high quality data from various orbiting spacecraft, such Wind, Stereo, Cluster, Themis, and the more recent one, the NASA Magnetospheric MultiScale (MMS) mission. In comparison with the solar wind, plasma turbulence in the magnetosheath remains far less explored, possibly because of the complexity of the magnetosheath dynamics that challenges any "realistic" theoretical modeling of turbulence in it. This complexity is due to different reasons such as the confinement of the magnetosheath plasma between two dynamical boundaries, namely the bow shock and the magnetopause; the high variability of the SW pressure that "shakes" and compresses continuously the magnetosheath plasma; and the presence of large density fluctuations and temperature anisotropies that generate various instabilities and plasma modes. In this paper we will review some results that we have obtained in recent years on plasma turbulence in the SW and the magnetosheath, both at the Magneto-HydroDynamics (MHD) and the sub-ion (kinetic) scales, using the state of the art theoretical models and in-situ spacecraft observations. We will focus on three major features of the plasma turbulence, namely its nature and scaling laws, the role of small scale coherent structures in plasma heating, and the role of density fluctuations in enhancing the turbulent energy cascade rate. The latter is estimated using (analytical) exact laws derived for compressible MHD theories applied to in-situ observations from the Cluster and Themis spacecraft. Finally, we will discuss some current trends in space plasmas turbulence research and future space missions dedicated to this topic that are currently being prepared within the community.
\end{abstract}

F. Sahraoui

Laboratoire de Physique des Plasmas, LPP/CNRS-Ecole Polytechnique-Sorbonne Universit-Paris SaclayObservatoire de Paris Route de Saclay, 91128 Ecole Polytechnique

Tel.: +331-6933-5922

E-mail: fouad.sahraoui@1pp.polytechnique.fr

S. Author

second address 
Keywords Plasma $\cdot$ Turbulence $\cdot$ solar wind $\cdot$ magnetosheath $\cdot$ in-situ data

\section{Introduction}

Turbulence is ubiquitous in fluid and plasmas flows [Frisch(1995), Goldstein et al.(1995),

Matthaeus \& Velli(2011)]. It plays a leading role in mass transport and energy (or other invariant quantities) transfers through scales until it is dissipated at the very small scales [Schekochihin et al.(2009) Howes et al.(2011)]. Unlike neutral fluids, ionized plasmas have often a mean magnetic field that affects significantly the turbulence properties. Indeed, non-zero mean field introduces: i) a specific direction in the plasma that violates the rotation symmetry and renders the turbulence spatially anisotropic; ii) characteristic scales of charged particles (e.g., electron and ion Larmor radii), where the property of scale invariance of the turbulence is broken. This is manifested in turbulence observations by the presence of spectral breaks in the energy spectra around those characteristic scales [Sahraoui et al.(2009)]. A third major difference exists between neutral fluids and collisionless (or weakly collisional) plasmas: the nature of the dissipation mechanisms and associated characteristic scales. In the former, dissipation is controlled by viscosity originating from molecular collisions originating at the microscopic level, while in the latter it is mediated by kinetic processes in the form of wave-particle interactions, such as Landau damping |Landau(1946) Howes et al.(2008), Schekochihin et al.(2009), Gary \& Smith(2009) Sahraoui et al.(2010a), Podesta et al.(2010), Sulem \& Passot(2015), Kobayashi et al.(2017)], cyclotron damping [Leamon et al.(1998), Kasper et al.(2008), Cranmer(2014), He et al.(2015)] and stochastic heating [Chandran et al.(2010)], which would involve different spatial or temporal scales. Often magnetic reconnection is evoked as a potential dissipation process in localized current sheets [Matthaeus et al.(1984), Retino et al.(2007)| Sundvist et al.(2007), Chasapis et al.(2015)]. However, magnetic reconnection and wave-particles interactions should not be seen as mutually exclusive. For instance, Landau damping is shown to be very effective in numerical simulations of collisionless magnetic reconnection [Tenbarge \& Howes (2013), Loureiro et al.(2013), Numata \& Loureiro(2015)].

Turbulence in astrophysical plasmas is a very active area of research because of the role that it plays in different objects. In the heliosphere, turbulence is thought to be responsible for the heating of the solar corona and for accelerating the solar wind (SW) [Matthaeus et al.(1984) Goldstein et al.(1995) Bruno \& Carbone(2005) Liu et al.(2006)]. In the interstellar medium (ISM), turbulence generated by supernovae explosions plays a role in star formation by preventing the collapse of selfgravitating molecular clouds (see [Schekochihin et al.(2009) Brandenburg et al.(2011) Federrath et al.(2016)] and the references therein). In accretion disks of black holes or neutron stars, turbulence is thought to be generated by the Magneto-Rotational Instability (MRI) that allows converting the gravitational potential energy of the inflowing mass into MHD turbulence at the outer scale (i.e., scale size of the disk height), which then cascades to smaller scales (following the classical picture of MHD cascade), where it is converted into heat [Balbus et al.(1998)]. The heat is observable through the energetic X-rays and radio emissions emitted by the disks. In tokamakcs, turbulence that can be generated by different plasma instabilities due to 
strong density and temperature gradients (e.g., Ion or Electron Temperature Gradient, ITG and ETG) is the major obstacle that prevents long-time plasma confinement [Diamond et al.(2005)].

\section{The near-Earth space plasmas}

The near-Earth space plasmas refer to the plasmas of the Earth's magnetosphere and the SW at $1 \mathrm{AU}$ (Astronomical Unit). Because of their accessibility to very detailed insitu measurements, these regions of space are an excellent laboratory for the study of different fundamental plasma processes. This is particularly true for fully-developed turbulence studies because the high Reynolds numbers $\left(\sim 10^{5}\right)$ estimated from spacecraft observations [Matthaeus et al.(2005), Bruno \& Carbone(2005)]. By in-situ measurements we refer to data taken by wave and particle instruments on board orbiting spacecraft. These instruments includes DC and AC magnetometers, electric field antennas, electron and ion mass and energy spectrometers that measure particle velocity distribution functions (VDFs) and plasma composition, which allows one to compute the plasma moments (density, velocity, temperature, ...) of each species. Often, energetic particles detectors and radio wave receivers are also available.

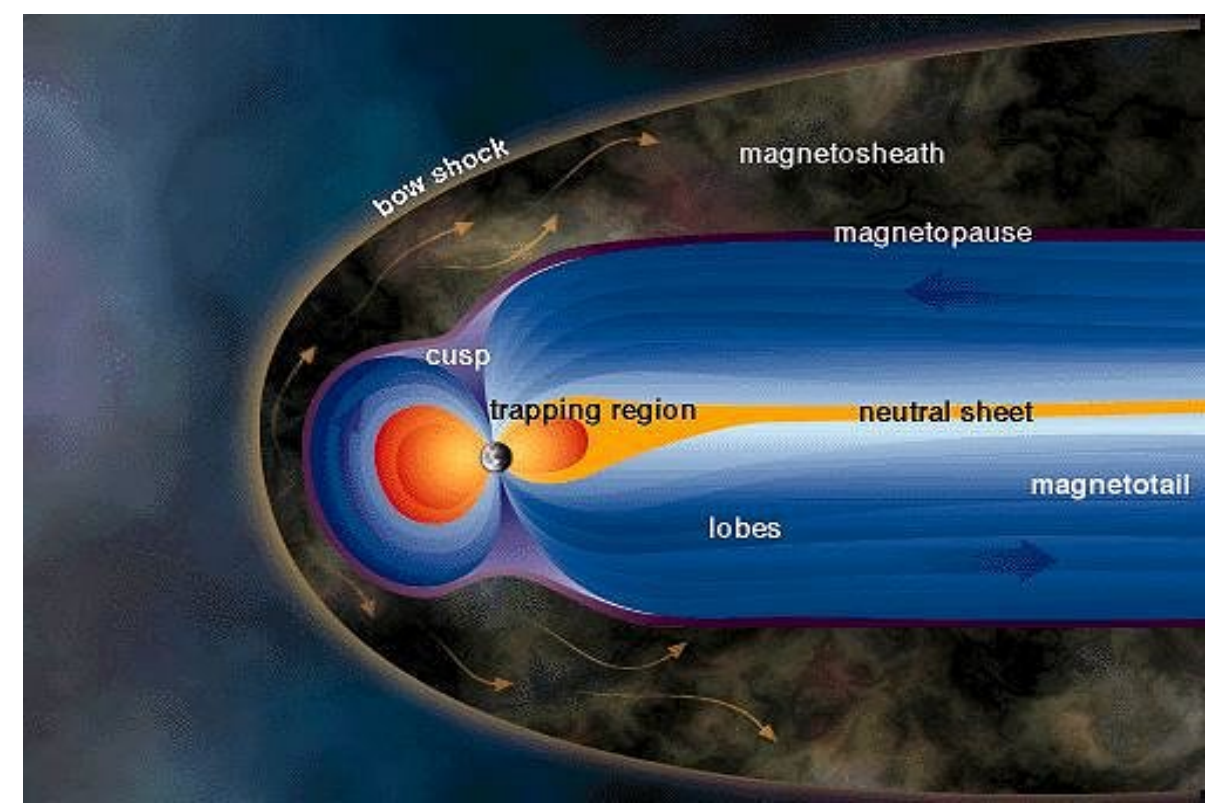

Fig. 1 A 2D view of the terrestrial magnetosphere showing its different key regions resulting from the interaction with the solar wind.

The results that we present in this paper were obtained in the SW and the magnetosheath, which is the region of space located behind the terrestrial bow shock 
(Figure 11). It is the interface of interaction between the SW and the magnetosphere, which makes the understanding of the physical processes occurring in it very crucial to apprehend the global SW-magnetosphere coupling. In particular, turbulence in the magnetosheath is believed to drive the processes of energy and particle transfers into the inner magnetosphere through the magnetopause [Belmont \& Rezeau(2001), Karimabadi et al.(2014)]. Due to the shock wave, the magnetosheath plasma is slower, hotter and denser than the SW plasma. Behind the quasi-parallel shock the magnetosheath plasma is known to be very turbulent with larger amplitude fluctuations than in the SW. From the observational viewpoint, the large amplitude of the turbulent fluctuations in the magnetosheath make them more accessible to wave instruments, in particular at small (kinetic) scales, because of their high SNRs (Signal-to-NoiseRatio) compared to those encountered in the SW [Sahraoui et al.(2013)].

Beyond the near-Earth space, the exploration of the inner heliosphere by missions such as the Parker Solar Porbe (PSP) [Fox et al.(2016)] and soon the Solar Orbiter mission [Muller et al.(2013)], or the planetary plasma environments (Mercury, Saturn, Jupiter) allows us to access a broad parameter space for which fundamental plasma processes can be studied (Fig. 2). This is crucial if one wants to extrapolate some of the results obtained in these media to remote astrophysical objects not accessible to in-situ measurements (e.g., the heliosheath, ISM or accretion disks) [Schekochihin et al.(2009), Vaivads et al.(2016), Verscharen et al.(2019)].

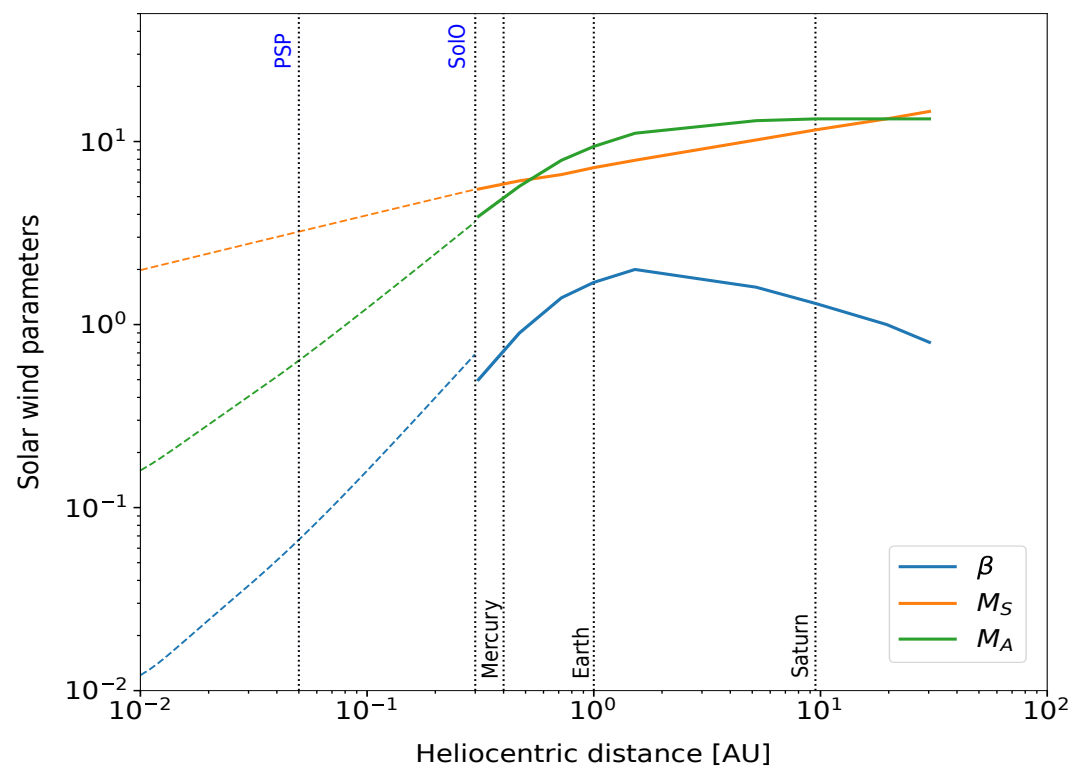

Fig. 2 Radial evolution of the the Alfvén $\left(M_{A}\right)$ and sonic $\left(M_{S}\right)$ Mach numbers and the plasma $\beta$ inferred from the models of [Hellinger et al.(2013)] and [Stverak et al.(2015)] for distances $<0.3 \mathrm{AU}$ (dahsed lines) and from [Slavin \& Holzer(1981)] for distances $>0.3 \mathrm{AU}$ (solid lines). The vertical dashed lines indicate average locations of the Saturn, Earth and Mercury, and the closest distance of the Solar Orbiter (SoLO) and Parker Solar Probe (PSP) spacecraft to the Sun. 


\section{Solar wind turbulence}

In the heliosphere, the solar corona and SW heating and acceleration are two major problems that resist a satisfactory explanation despite decades of intensive research work [Bruno \& Carbone(2005)]. The main difficulty stems from the (nearly) collisionless nature of the SW plasma: the mean free-path of particles is $\sim 1 \mathrm{AU}$. Therefore, fluid viscosity and electric resistivity that originate from particle collisions at the molecular level are virtually absent. Thus, a fundamental question emerges: how to explain the observed heating or acceleration of the plasma particles, often to relativistic energies [Matthaeus et al.(1984), Liu et al.(2006) Cranmer(2014), Matthaeus \& Velli(2011)]?

How is energy that originates from the macroscales dissipated at the microscales? Turbulence is a crucial ingredient to explain these processes: it allows for transporting (free) energy injected at the largest scales of the system to smaller scales where kinetic processes can transfer it to the plasma particles [Schekochihin et al.(2009), Sahraoui et al.(2009), Howes et al.(2011)].

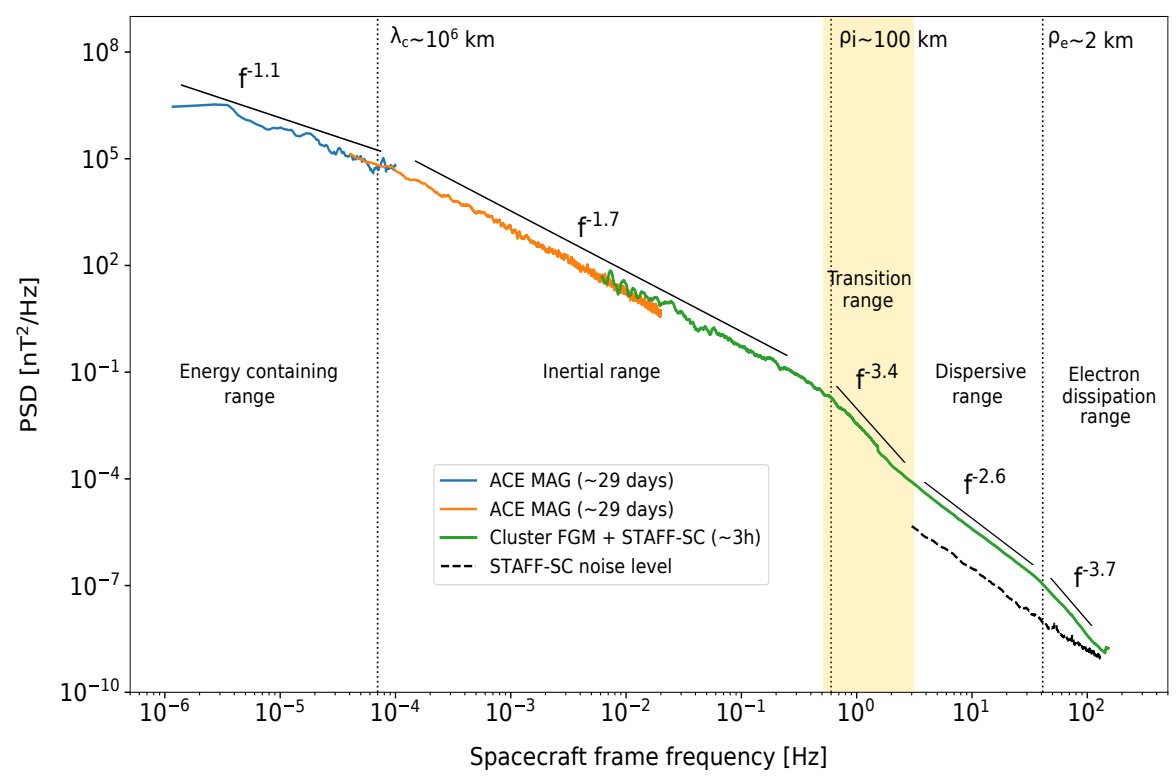

Fig. 3 A magnetic energy spectrum measured in the solar wind by ACE and Cluster spacecraft, depicting a cascade over 8 decades in frequency, from the large MHD scales $\left(\sim 10^{5}-10^{6} \mathrm{~km}\right)$ down to the electron scales $(\sim 1 \mathrm{~km})$. The spectrum shows different frequences band and slopes discussed in the text. The vertical dashed lines indicate the frequencies corresponding to Taylor shifting the correlation scale $\lambda_{C}$, and the ion $\left(\rho_{i}\right)$ and electron $\left(\rho_{e}\right)$ Larmor radii.

Indeed, in-situ observation in the SW showed that the power spectral density (PSD) of the magnetic field fluctuations covers a wide range of scales, from macroscopic scales, where the MHD approximation is valid, to microphysical scales of the order of the Larmor radius of charged particles where kinetic effects become impor- 
tant [Coleman(1968), Matthaeus \& Goldstein(1982) Leamon et al.(1998)]. Unlike in fluid turbulence [Frisch(1995)], the observed magnetic PSDs in the SW exhibit distinct frequency bands that span at least eight decades of frequencies and that have power-laws with different exponents. As example is shown in Figure 3 , which was obtained in the SW by combining data from the ACE spacecraft (from 2019-0609 02:00 to 2019-07-08 18:00) and the Cluster spacecraft (2006-03-19 from 20:30 to 23:20). The spectrum shows five spectral bands separated by breaks that occur near the correlation length scale $\left(\lambda_{C}\right)$ of the turbulence and the characteristic scales (gyroradii or inertial lengths) of ions and electrons. First is the $f^{-1}$ range generally observed in the fast SW for frequencies $\lesssim 10^{-4} \mathrm{~Hz}$ (given in the spacecraft reference frame - see below the discussion about converting observed frequencies into wavenumber). The origin of this range, first reported in [Russell (1972)] and often referred to as the energy containing scales [Matthaeus et al.(1994)] or the " $1 / f$ flicker noise" [Matthaeus et al.(1986)], remains an open question [Chandran (2018) Matteini et al.(2018)]. It includes the superposition of magnetic elements with different statistical properties that emerge from the corona due to magnetic reconnection [Matthaeus et al.(1986)], evolution of the Alfvén waves coming from the corona and their reflection in the expanding SW [Velli et al.(1989).Verdini et al.(2012)] and inverse cascade in MHD turbulence [Dmitruk et al.(2007)]. It is worth recalling that the "1/f noise" spectrum is observed also in planetary magnetosheaths |Hadid et al.(2015), Huang et al.(2017a)], in the solar photospheric magnetic field [Matthaeus et al.(2007)] and in a variety of other systems such as electronic devices, dynamo experiments and geophysical flows (see [Dmitruk et al.(2011)] and the references therein). Second is the inertial range with a scaling $f^{-5 / 3}$ in the frequency range $\sim\left[10^{-4}, 10^{-1}\right] \mathrm{Hz}^{1}$ This range, where dissipation is assumed to be negligible [Kolmogorov(1941)], is thought to be generated via nonlinear interactions between counter-propagating incompressible Alfvén wave-packets [Iroshnikov(1963), Kraichnan(1965)]. These interactions generate smaller and smaller structures, hence the classical paradigm of energy cascade from large to small scales. Third is the range near the ion characteristic scales $\sim[0.1,1] \mathrm{Hz}$ that we call the transition range [Sahraoui et al.(2010a)] (it is often referred to as the dissipation range), where spectra can steepen significantly to $\sim f^{-4.5}$ [Goldstein et al.(1994), Leamon et al.(1998), Stawicki et al.(2001), Smith et al.(2006), Sahraoui et al.(2010a) Bruno et al.(2014)]. The actual scaling and the physics in this zone is still an unsettled question, e.g., [Bruno et al.(2014). Voitenko \& De Keyser(2016) Kobayashi et al.(2017). Passot \& Sulem(2019)]. Fourth is the dispersive range far below the ion scale, $\sim[3,30] \mathrm{Hz}$, with a scaling $f^{\alpha}$ and $\alpha \in[-3.1,-2.3]$ [Sahraoui et al.(2009), Kiyani et al.(2009). Alexandrova et al.(2012), Sahraoui et al.(2013)]. In this range, where dispersive and dissipation effects become important [Schekochihin et al.(2009), Passot \& Sulem(2015)], the nature of the turbulent fluctuations is also an unsettled question [Bale et al.(2005), Sahraoui et al.(2009), Sahraoui et al.(2010a), Podesta et al.(2012b), Gary et al.(2012), He et al.(2011) |He et al.(2012), Salem et al.(2012), Kiyani et al.(2013)], although there is an increasing body of evidence that the cascade is dominated by the Kinetic Alfvén Mode (KAW) rather than the popular whistler mode [Sahraoui et al.(2010a), Podesta et al.(2012b),

\footnotetext{
1 There is still a debate as to whether the scaling of the PSDs of the magnetic and the velocity fluctuations this range follow the Kolmogorov spectrum $k^{-5 / 3}$ or the IK prediction $k-3 / 2$. This point will not be further discussed in this paper.
} 
Salem et al.(2012) Podesta(2013)]. Fifth is the electron dissipation range $((f>40$ $\mathrm{Hz}$ ) where a new steepening of the magnetic energy spectra has been reported and was interpreted as an ultimate dissipation of the residual magnetic energy into electron heating [Sahraoui et al.(2009)]. Due to instrumental limitations, the actual scaling of the magnetic energy spectra at sub-electron scales remains an open question in SW turbulence [Alexandrova et al.(2012), Sahraoui et al.(2013)].

Although SW turbulence has been a subject of intensive theoretical, numerical and observational research work over the past decades, many questions remain open. Examples of these questions are:

- What are the actual scaling laws, e.g. $\delta B^{2}\left(k_{\|}, k_{\perp}\right) \sim k_{\|}^{-\alpha_{1}} k_{\perp}{ }^{-\alpha_{2}}$ that are "hidden" behind an observed spectrum onboard spacecraft $\delta B^{2}(f) \sim f^{-\alpha}$ ? How to measure accurately the anisotropy of turbulence in wavenumber with respect to the mean magnetic field (i.e., $k_{\|} v s \quad k_{\perp}$ )?

- What are the actual plasma modes involved in the turbulence cascade at kinetic scales? How can we identify them unambiguously? How do the plasma kinetic instabilities fit into the global picture of fluid-like cascade?

- What are the actual mechanisms of energy "dissipation" into particle heating or acceleration? What are their signatures in velocity space? How to identify them in spacecraft data?

- What role coherent structures may play in the turbulence cascade and energy dissipation?

The first question above points toward a tricky problem is studies of turbulence using spacecraft data that warrants a short discussion here. This problem is commonly referred to as the spatio-temporal ambiguity and is inherent to the interpretation of any measurement made onboard a single spacecraft. In turbulence studies the problem is better reflected in the difficulty to infer the wavenumber spectra (generally predicted by turbulence theories [Galtier (2006), Meyrand \& Galtier(2010), Schekochihin et al.(2009) Boldyrev \& Perez(2012)]) from the frequency ones measured onboard the spacecraft (Fig. 3). Indeed, each frequency $\omega_{s c}$ in that spectrum has two contributions, a temporal one coming from the frequency of the fluctuation in the plasma rest frame $\omega_{\text {plas }}$, and a spatial one coming from the Doppler shift $\mathbf{k}$.V. These three quantities are related by Eq. 1 .

$$
\omega_{s c}=\omega_{\text {plas }}+\mathbf{k} \cdot \mathbf{V}=\omega_{\text {plas }}+k V \cos \theta_{k V}
$$

where $\theta_{k V}$ is the angle between wave vector $\mathbf{k}$ and the flow speed $\mathbf{V}$.

In the solar wind, because the phase speeds of the fluctuations in the inertial range are much smaller than the flow speed $\left(V_{A} \sim C_{S}<<V\right.$, where $V_{A} \sim C_{S} \sim 100 \mathrm{~km} / \mathrm{s}$ is the Alfvén and sound speeds), the Taylor hypothesis is generally used to infer the spectra in wavenumber space from the frequency ones measured onboard the spacecraft ${ }^{2}$ However, one should keep in mind that even when the Taylor hypothesis is valid [Howes et al.(2014), Perri et al.(2014)], it provides wavenumber spectra only along the plasma flow direction $\left(\omega_{\text {plas }}<<\mathbf{k} \mathbf{V} \Rightarrow \omega_{s c} \sim \mathbf{k} \mathbf{V}=k V \cos \theta_{k V}\right)$. The two other

\footnotetext{
2 In fact, the phase speeds must be smaller than the flow speed projected onto the wave vector $\mathbf{k}$ as can be seen in $\mathrm{Eq} 1$
} 
directions perpendicular to the mean flow remain thus unresolved. Furthermore, in the dispersive range the phase speeds of the turbulent fluctuations are expected to increase as function of the wavenumber [Galtier (2006) Sahraoui et al.(2007), Howes et al.(2011), Boldyrev et al.(2013)]. This is true at least for the KAW and whistler modes, the two main channels debated in the literature that would carry the energy cascade down to the electron scales. To unambiguously determine $\omega_{\text {plas }}$ it is necessary to estimate the k-spectrum at each observed frequency $\omega_{\text {plas }}$, which requires using multi-spacecraft dat 3 and appropriate techniques such as the k-filtering technique (known also as the wave telescope [Sahraoui et al.(2003), Tjulin et al.(2005), Sahraoui et al.(2010b), Narita et al.(2010a), Narita et al.(2010b), Huang et al.(2010) Roberts et al.(2017)]. Note that these constraints hold also for the magnetosheath plasmas, possibly even in a more stringent way, since even at large (MHD) scales the characteristic phase speeds in the medium become comparable to the flow speed $\left(V_{A} \sim C_{S} \sim V \sim 200 \mathrm{~km} / \mathrm{s}\right)$. This is because of the deceleration of the SW flow downstream of the bow shock.

In the absence of multispacecraft data, often one has no choice other applying the Taylor hypothesis. This has been done in observational studies of both MHD and subion scales turbulence [Bale et al.(2005), Sahraoui et al.(2009), Alexandrova et al.(2012)], although its validity at those small scales can be questioned. To improve those studies [Sahraoui et al.(2012)] proposed a test of the validity of the Taylor hypotheis that can be achieved on single spcacecraft data, which is based on the estimation of the ratio between the spectral breaks at electron and ions scales. The test has been applied recently to Cluster data in the SW and showed that a fraction of the analyzed data sample can indeed violate the Taylor hypothesis at electron scales [Huang \& Sahraoui(2019)]. Note finally that other recent papers have raised the possibility of violating of the Taylor hypothesis by MHD scales fluctuations taken in the inner heliosphere as those expected from the PSP mission [Matthaeus et al.(2016), Bourouaine \& Perez(2018)].

\section{Magnetosheath turbulence}

In comparison with SW turbulence, the magnetosheath turbulence is much less investigated. This might be due to the complexity of the magnetosheath plasma (as explained above), which renders the use of popular turbulence models (e.g. incompressible MHD widely used in SW studies) inappropriate for the magnetosheath plasma. For this reason we will focus in the paper on reviewing some the results that we have obtained on magnetosheath turbulence using in-situ data from the Cluster and Themis spacecraft, but still pay attention to comparing them to their counterparts in the SW to highlight their major common features and differences. We emphasize here that this paper is not a formal review that would cover all the work done by the community in recent years. It is rather a collection of some of our own results and some of our personal views of these challenging problems of space plasmas, as clearly stated in the

\footnotetext{
3 More rigorously, estimating $\omega_{\text {plas }}$ requires only measuring one component of the k-vector, namely component parallel to the flow $\mathbf{V}$. However, once $\omega_{\text {plas }}$ is determined the full $\mathbf{k}$-vector is needed to determine $3 \mathrm{D}$ dispersion relations, i.e., $\omega_{\text {plas }}=\omega_{\text {plas }}(\mathbf{k})$.
} 
title of the paper. The paper is intended to a broader audience of plasmas physicists beyond the space physics community.

A few studies have been carried out in the past on magnetosheath turbulence [Sahraoui et al.(2003), Sahraoui et al.(2006), Lacombe et al.(2006), Mangeney et al.(2006), Alexandrova et al.(2008), Yordanova et al.(2008), He et al.(2011)]. Two main similarities with SW turbulence emerged from those studies: i) A strong anisotropy $\left(k_{\|}<<\right.$ $k_{\perp}$ ) both at sub-ion and electron scales [Sahraoui et al.(2006), Mangeney et al.(2006), Chen et al.(2017)], ii) The presence of kinetic instabilities and nonlinear structures [Sahraoui et al.(2004). Sahraoui et al.(2008), Alexandrova et al.(2006)]. Major differences exist though, e.g., i) magnetosheath turbulence evolves in a "confined" region of space bounded by the bow shock and the magnetopause; these boundaries may influence the anisotropy of the turbulence [Sahraoui et al.(2006) Yordanova et al.(2008)]; ii) the dominance of very low frequency compressible fluctuations such as the mirror modes [Horbury et al.(2004). Sahraoui et al.(2006)]. However, large statistical surveys of the turbulence properties in the magnetosheath have been carried out only in recent years. We will now review some of the reported results and compare them with SW turbulence.

\subsection{Spectral laws in the MHD range}

Owing to the fact that the magnetosheath plasma evolves in a confined medium limited by the bow shock and the magnetopause (Figure 1), turbulence properties, at least at large scales, are expected to depend on the dynamical pressure of the SW, the location within the magnetosheath or the geometry (quasi-parallel and quasiperpendicular) of the bow shock [Karimabadi et al.(2014)]. Analyses of large (MHD) scales turbulence in the magnetosheath of Saturn and Earth showed interesting features that are quite different from those known in the SW [Hadid et al.(2015), Huang et al.(2017a)]. Two examples of the observed spectra are shown in Figure 4 Intriguingly, some of the spectra showed no inertial range with the so-called Kolmogorov scaling $f^{-5 / 3}$ : Turbulence transitions from the $\sim f^{-1}$ spectrum to $\sim f^{-2.5}$ at kinetic scales, while other spectra were found to have an inertial range [Alexandrova et al.(2008)]. The breaks observed occur near the ion characteristic scales $\rho_{i}$ or $d_{i}$. The $f^{-1}$ spectrum at MHD scales recalls the spectrum of the energy containing scales in SW turbulence [Bruno \& Carbone(2005)].

To explain the absence of the Kolmogorov inertial range we conducted a large statistical study using more than 3 years of the Cluster data, which involved different orbits and different locations of the magnetosheath [Huang et al.(2017a)|. A large fraction of the spectra did not show the Kolmogorov inertial range. When we investigated the distribution of the observed slopes at different locations in the magnetosheath we clearly evidenced that the Kolmogorov-like inertial range occurs essentially on the flanks of the magnetosheath away from the bow shock (Figure 5). This observation questions the universality of the Kolmogorov spectrum and the existence of the inertial range in magnetosheath plasma. At kinetic scales the slopes do not seem to depend on the location within the magnetosheath. This may be explained by the fact that the scales involved $\left(l \leq 10^{2} \mathrm{~km}\right)$ are much smaller than the scale size 

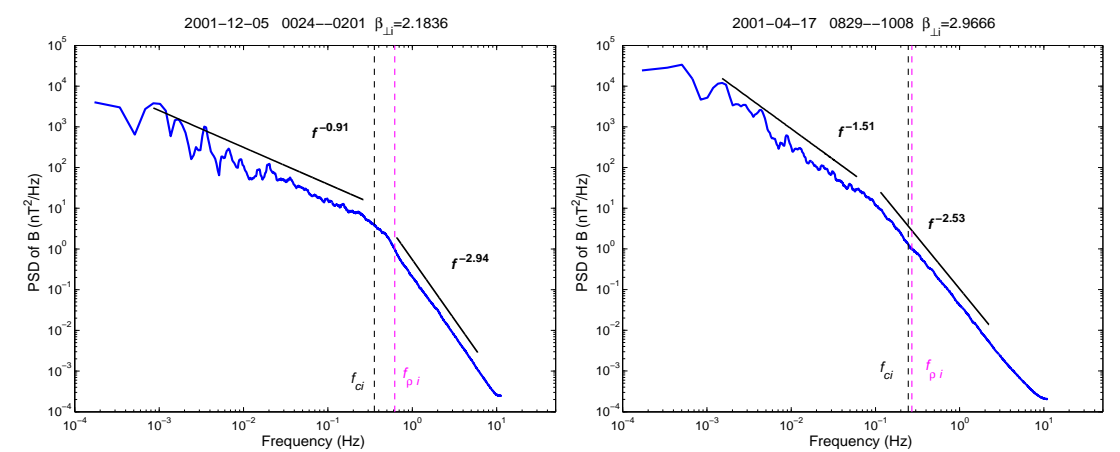

Fig. 4 Examples of spectra measured in the magnetosheath showing the absence of the Kolmogorov inertial range (left) and its presence (right). The vertical dashed lines indicate the ion gyrofrequency $f_{c i}$ and Taylor-shifted Larmor scale $f_{\rho_{i}}$.

of the magnetosheath $\left(\sim 10^{5} \mathrm{~km}\right)$. In other words, the turbulence at kinetic scales is "far" from the outer scales represented by the boundaries of the magnetosheath.

An immediate question that emerges from the previous observations is the reason of the absence of the Kolmogorov inertial range behind the bow shock. In [Huang et al.(2017a)] we argued that it may be caused by the interaction between the SW and the shock itself. The shock somehow "destroys" the pre-existing correlations between the turbulent fluctuations in the SW, which causes phase randomization. We verified indeed this feature at large scales (i.e., above the ion spectral break) and showed that the fluctuations are quasi-Gaussian [Hadid et al.(2015)] (see also [Wicks et al.(2013)] about the "nonturbulent" nature of the $1 / f$ spectrum). Once those random-like fluctuations are generated, they evolve in time and may interact nonlinearly as they propagate (or get advected) away from the shock. The state of fully developed turbulence is reached only away from the shock where the Kolmogorov inertial range is observed. This scenario has been verified experimentally by comparing the estimated correlation time of the turbulent fluctuations and their travel time within the magnetosheath as they get advected by the flow [Huang et al.(2017a)].

Another question that has been explored was the nature of the fluctuations populating the inertial range. Are they Alfvénic, as in the SW, or compressible magnetosonic? To answer these questions we used the magnetic compressibility $C_{\|}$given by the ratio between the PSDs of the parallel and total magnetic field fluctuations (parallel is w.r.t. the background magnetic field $B_{0}$ ) [Gary \& Smith(2009), Salem et al.(2012)]:

$$
C_{\|}(f)=\frac{\left|\delta B_{\|}(f)\right|^{2}}{\left|\delta B_{\|}(f)\right|^{2}+\left|\delta B_{\perp}(f)\right|^{2}}
$$

where $\delta B(f)^{2}$ is the PSD of the magnetic fluctuations. From linear kinetic theory, the Alfvén and the magnetosonic modes are known to have very different profiles of the magnetic compressibility [Sahraoui et al.(2012)]. Briefly speaking, one can say that at MHD scales Alfvénic fluctuation [Goldstein et al.(1995)] have $C_{\|}<<1$ while compressible magnetosonic fluctuations have $C_{\|} \gtrsim 0.3$. Therefore, magnetic 

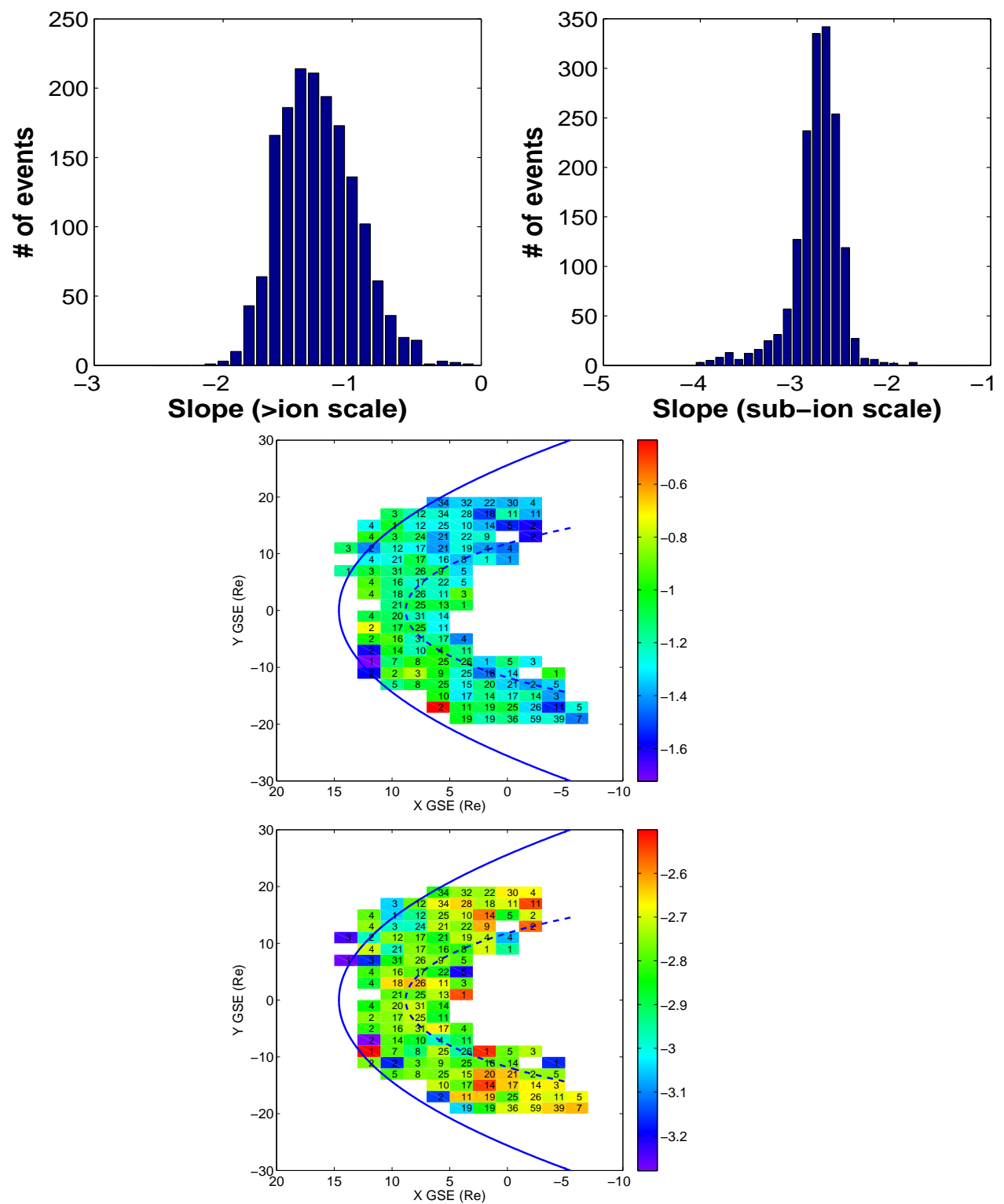

Fig. 5 Top: The histogram of the measured spectral slopes obtained from a survey of 3 years Cluster data in the magnetosheath at MHD scales (left) and sub-ion scales (right). Bottom: The distribution of the slopes within the magnetosheath. The $\sim f^{-1}$ spectra are more observed on the sub-solar point near the shock, while the Kolmogorov spectrum $\sim f^{-5 / 3}$ is observed more in the flanks of the magnetosheath. The numbers given in each pixel represent the number of the analyzed spectra. The vertical color bar gives the values of the spectral slopes (adapted from [Huang et al.(2017a)]). 
compressibility can be easily used to check the dominance (or not) of the Alfvénic fluctuations in our data [Podesta et al.(2012a) Kiyani et al.(2013)]. However, there is a caveat regarding the computation of $C_{\|}$when the magnetic fluctuations are high and become comparable to the mean field $B_{0}$ (i.e., $\delta B / B_{0} \sim 1$ ). In this case the parallel direction is ill-defined if the global (mean) field is used to project onto it the magnetic fluctuations. Instead, a local field decomposition is necessary to capture correctly the parallel and perpendicular fluctuations. A method based on a scale-by-scale decomposition is developed and used in [Kiyani et al.(2013)].
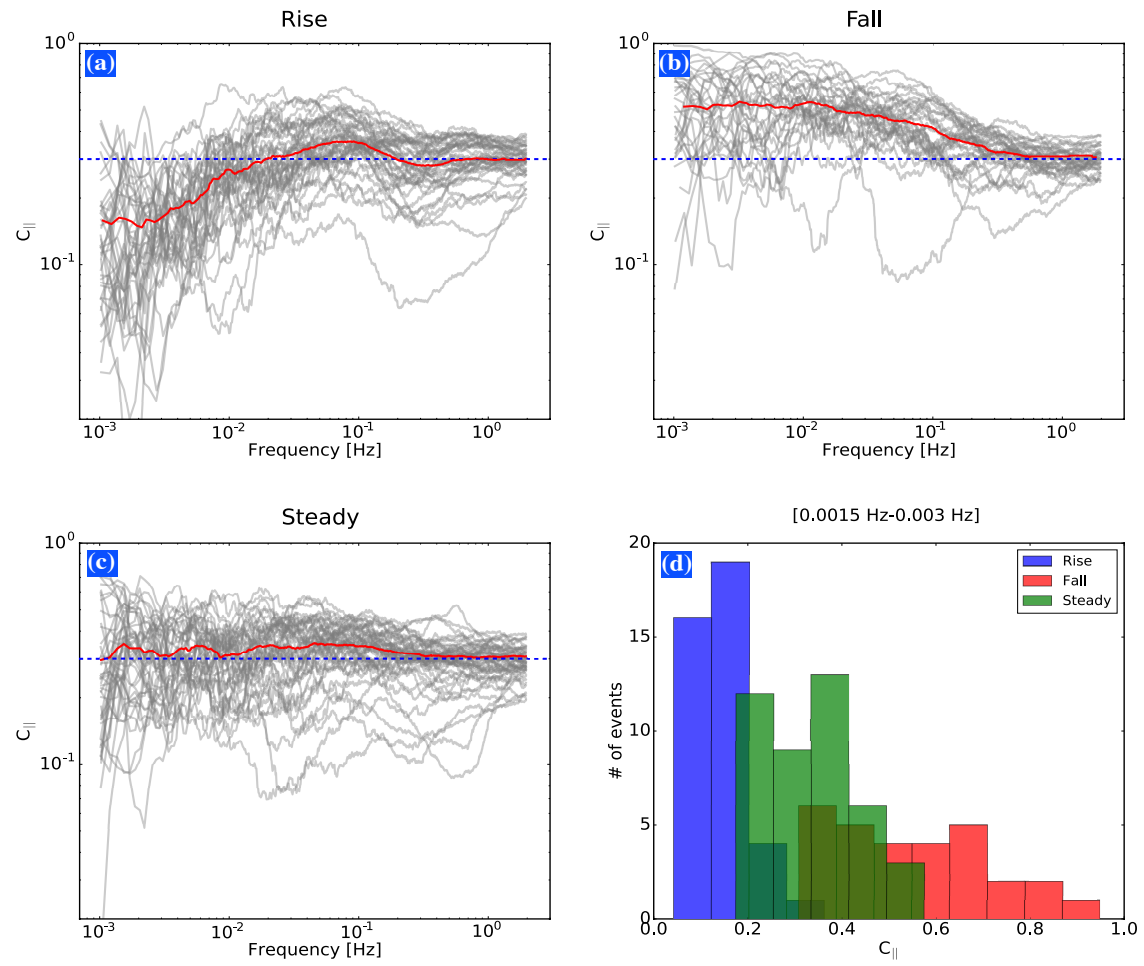

Fig. 6 Estimated magnetic compressibility $C_{\|}$for all statistical events that have a Kolmogorov-like scaling. Three distinct profiles were observed (grey curves: all events, red curves: mean values): (a) rising characteristic of shear Alfvén wave turbulence, (b) falling-off and (c) steady, both characteristic of compressible magnetosonic-like dominated turbulence. The dashed blue lines in (a-c) indicate the value $1 / 3$ of the compressibility. (d) shows the histogram of $C_{\|}$iaveraged over the frequency range $[0.004,0.1] \mathrm{Hz}$.

The results of the analysis are shown in Figure 6 . Different profiles of $C_{\|}$reflect different nature of the turbulent fluctuations [Huang et al.(2017a)]. The main result though is that only a fraction (35\%) of the observed Kolmogorov spectra were populated by shear Alfvénic fluctuations, whereas the majority of the events $(65 \%)$ was found to be dominated by compressible magnetosonic-like fluctuations, which con- 
trasts with well-known turbulence properties in the SW. This results shows that, unlike the SW, density fluctuations are important at the MHD scales in magnetosheath turbulence, and must be considered in theoretical modeling of the magnetosheath plasma.

\subsection{Spectral laws at kinetic (sub-ion) scales}

As mentioned above, investigation of sub-ion and electron scale turbulence in the SW faces strong limitations due to the low amplitude electric and magnetic field fluctuations, which require very high sensitivity instruments to capture them. In the magnetosheath these restrictions can generally be relaxed considering the increase of the amplitude of the turbulent fluctuations across the bow shock. This somehow relaxes the stringent requirement on the wave instruments sensitivity when it comes to probing into the magnetosheath plasma. Another advantage of the magnetosheath is the availability of a huge amount of high quality data that covers a broader range of physical parameters compared to the SW at $1 \mathrm{AU}$ (see for instance the histograms of $\beta$ in [Sahraoui et al.(2013), Huang et al.(2014)]). This is particularly true after the successful launch of the MMS mission in 2015, which has been collecting data plasma data with unprecedented high time resolution.

As we showed in section 4.1. MHD scale turbulence in the magnetosheath shows features (e.g., the absence of the inertial range in the sub-solar region) that contrast with those in the SW. This has motivated a new study to investigate the universality of the turbulence scaling at the sub-ion and electron scales in the magnetosheath. To do so, we surveyed a large statistical sample of the Cluster/STAFF-SC waveforms (sampled at 225Hz) measured in the magnetosheath from 2002 to 2005, and compared the spectra of the magnetic energy above and below the electron break frequency [Huang et al.(2014)]. Some similarities and also differences were found with SW turbulence.

A similarity is found regarding the slopes in the dispersive range, i.e. between the ion and electron spectral breaks: the distribution is narrow and peaks near -2.8 (see Figure 7). Considering the differences in the plasma parameters in the two regions, which may drive different plasmas modes and instabilities, this result is rather surprising and suggests that the scaling of turbulence does not depend statistically on the local plasma conditions. This has been confirmed by the absence of significant correlation between those slopes and the local plasma parameters (e.g., the plasma $\beta$ ) [Sahraoui et al.(2013). Huang et al.(2014)]. A major difference was found in the histogram of the slopes below the electron spectral break (Figure 7). In the magnetosheath the slopes were found to be generally steeper: the distribution peaks near -5.5 and slopes as steep as -7 were observed. We suggested an explanation of this discrepancy in [Huang et al.(2014)] based on the differences in the SNRs between the two regions. Indeed, a good anti-correlation was found between the SNRs (computed at $\left.f=30 \mathrm{~Hz} \sim k \rho_{e} \sim 0.5\right)$ and the observed slopes : the higher is the SNR the steeper is the spectrum (see Figure 5 in [Huang et al.(2014)]). To show the effect of the low SNR we over-plotted in Figure 7 the histogram of the slopes for the magnetosheath events that have $\mathrm{SNR} \leq 10$ to mimic $\mathrm{SW}$ observations. The resulting new histogram, 

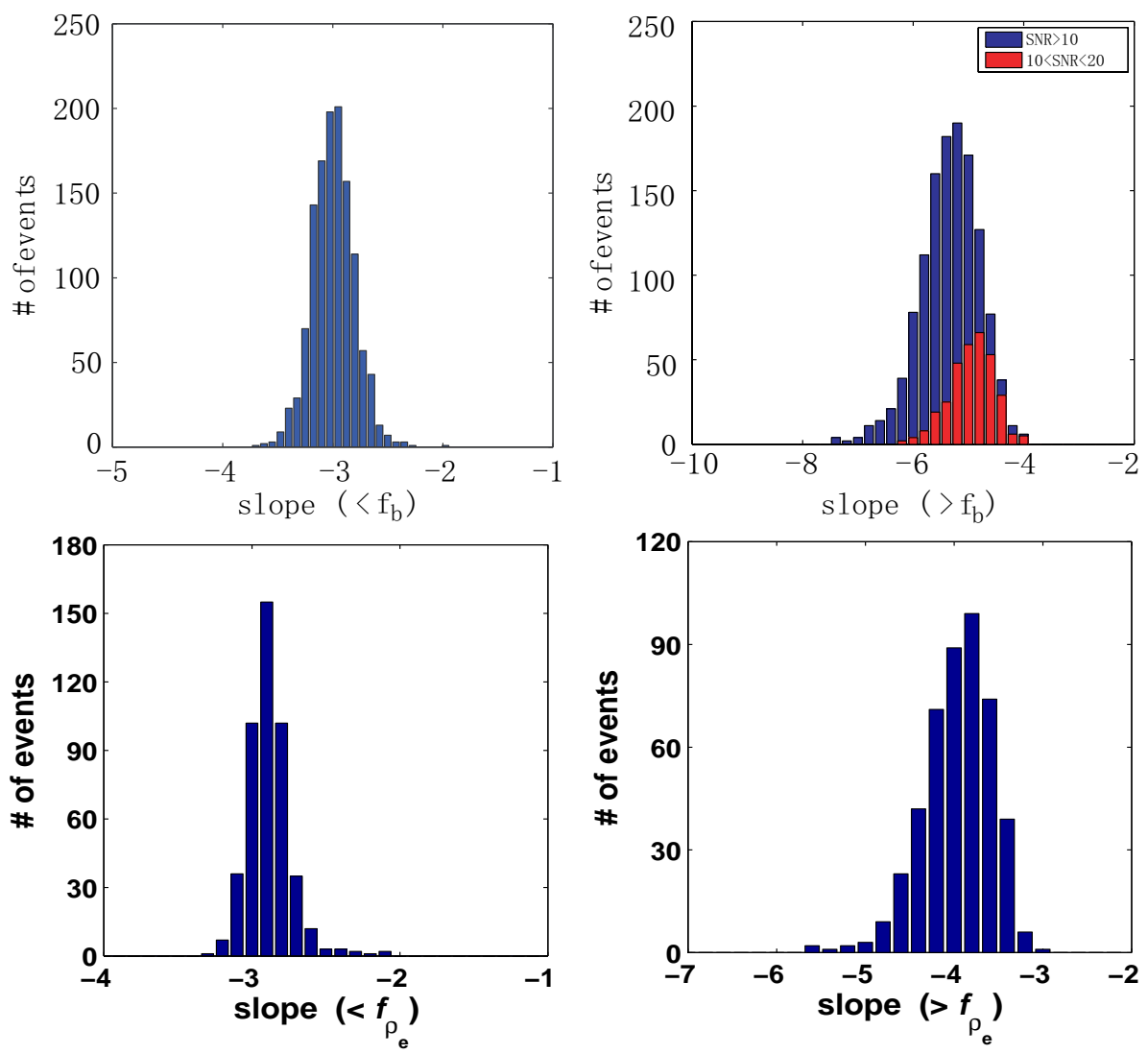

Fig. 7 Comparison between the scaling of the magnetic energy spectra below and above the electron spectral break, as observed in the magnetosheath (top, adapted from [Huang et al.(2014)|) and in the solar wind (bottom, adapted from [Sahraoui et al.(2013)]).

plotted in red in Figure 7, resembles quite well that of the SW. This finding suggests that an accurate determination of the turbulence scaling at, and below, the electron scale in the SW requires SNRs as high as those in the magnetosheath $(>25)$, for which the correlation SNR-slopes drops to zero (Figure 5 in [Huang et al.(2014)]). This requirement cannot be met with the current space missions in the SW at 1AU (see section 7) about the design of futures space missions with high sensitivity instruments dedicated to electron scale turbulence at $1 \mathrm{AU})$. In the inner heliosphere $(<0.3$ $\mathrm{AU}$ ), because of the increasing amplitude of the magnetic fluctuations the magnetometers onboard Solar Orbiter and the Parker Solar Probe missions would not face these limitations.

One of the striking observations that can be made from Figure 7 is that the distribution of the slopes, both in the SW and in the magnetosheath, become broader as we go to small scales, from the inertial range (see Figure 7] n [Smith et al.(2006)] $)$ to the electron scales (Fig. 7). After several attempts to explain this broadening based 
on energy dissipation via Landau damping and possible dependence on the nonlinearity parameter using Landau-fluid simulations[Kobayashi et al.(2017)], we recently proposed a rather simpler explanation based on the effect of violation of the Taylor hypothesis at electron scales. Using a simple toy model of linear dispersive KAW and whistler modes we succeeded in reproducing the observations of Figure 7 by transforming into the spacecraft frame a single power-law wavenumber spectrum with a scaling $k^{-2.8}$ using different flow and phase speeds of the fluctuations typical of the SW [Sahraoui \& Huang(2019)].

There are two open questions that are not addressed here. The first is very fundamental and is related to the origin of the sub-ion scale turbulence observed even when inertial range turbulence is absent. Indeed, the conventional wisdom holds that kinetic scale turbulence originates from the cascade taking place in the inertial range and transitioning into the sub-ion scales. Theoretically or numerically, this is modeled by the extension of Alfvénic turbulence into the kinetic scales, for example by including small scales effects such as the Hall term, electron inertia or FLRs (Finite Larmor radius effects) in fluid descriptions [Galtier (2006) Sulem \& Passot(2015), Andrés et al.(2016), Andrés et al.(2018a)], leading to the KAW or whistler turbulence models. However, when inertial range cascade is absent (or suppressed) a paradigm shift is needed, and other mechanisms must act to generate the observed fully developed kinetic turbulence. This might happen through ion scale instabilities (e.g., mirror or firehose) that may drive the sub-ion scale turbulence[Sahraoui et al.(2006), Kunz et al.(2014)], or magnetic reconnection as shown recently in Hybrid-Vlasov simulations [Cerri \& Califano(2017) Franci et al.(2017)]. This question requires further investigation.

The second question that is not discussed in detail in this review is the nature of the sub-ion scale turbulence, and the mechanisms by which energy is dissipated at those scales into ion and/or electron heating, whether or not turbulence at MHD scales is developed. As said earlier, there is now an ample evidence that KAW turbulence is likely to be the dominant component of turbulence at the sub-ion scales, at least in the limit $\beta_{i} \gtrsim 1$ [Sahraoui et al.(2010a). Podesta et al.(2012a). Salem et al.(2012), Podesta(2013) Cerri et al.(2017b)], while other minor components can be present such as ion cyclotron [He et al.(2015)], Bernstein [Sahraoui et al.(2012) Podesta et al.(2012b)] or whistler [Lacombe et al.(2014)] modes. However, there is no large statistical surveys that would confirm this conclusion. The reason is the absence of a simple diagnosis that can discriminate between the modes at those scales on which such statistical studies could be performed. For instance, the magnetic compressibility becomes not very useful at those scales because of power isotropisation [Sahraoui et al.(2012), Podesta et al.(2012a), Kiyani et al.(2013)]. Moreover, other complications come into play, for example, the possible violation of the Taylor hypothesis [Huang \& Sahraoui(2019)], the presence of plasma instabilities, instruments limitations or calibration issues. Overall, one can say that the interplay between sub-ion scale cascade, plasma instabilities, coherent structures, and magnetic reconnection is a vast and a poorly understood problem, which requires further investigation. 


\section{Energy cascade rate of compressible isothermal MHD turbulence}

Derivation of the so-called exact laws in plasma turbulence theories has attracted a particular attention over the past years. This is because these laws allow one to better understand the turbulence dynamics, to evidence turbulence inertial range in numerical and experimental data and to estimate the energy cascade rate in turbulent flows. The original idea developed by Kolmogorov [Kolmogorov(1941)] for Navier-Stokes equations was generalized to plasmas described within various theoretical frameworks: incompressible MHD (IMHD) [Politano \& Pouquet(1998)] (hereafter PP98), incompressible Hall-MHD (IHMHD) Galtier (2008), Banerjee \& Galtier(2017) Hellinger et al.(2018), Ferrand et al.(2019)], compressible MHD (CMHD) [Banerjee \& Galtier(2013)] (hereafter BG13) (see also the new derivation of the same law using the classical variables $\mathbf{v}, \mathbf{B}$ and $\rho$ instead of the Elsässer variables in [Andrés \& Sahraoui(2017)]), compressible Hall-MHD [Andrés et al.(2018a)] and incompressible two-fluid model [Andrés et al.(2016)]. The PP98 model has been widely applied to in-situ measurements in the SW to estimate the amount of the energy that is cascaded from large-tosmall scale where it is expected to be dissipated into plasma heating [Smith et al.(2006), Sorriso-Valvo et al.(2007) MacBride, Smith \& Forman(2008) Marino et al.(2008) Carbone et al.(2009). Smith et al.(2009), Stawarz et al.(2009) Osman et al.(2011), Coburn et al.(2014), Banerjee et al.(2016)]. The use of the PP98 model is justified because the SW is only weakly compressible $(\delta \rho / \rho \sim 20 \%$ [Hadid et al.(2017)] $)$. However, and as shown above, the terrestrial magnetosheath (and other planetary magnetosheaths [Hadid et al.(2015)] ) show higher density fluctuations $(\delta \rho / \rho \sim 50-100 \%$ [Sahraoui et al.(2006)]). Those observations highlighted the need for deriving more general exact laws of turbulence that include density fluctuations.

Here, we briefly review some of the results that we have obtained on estimating the compressible energy cascade rate in the SW and in the magnetosheath with an attempt to highlight the main differences and similarities between the two regions.

\subsection{Theoretical models}

We recall the basic equations of the two theoretical models, namely PP98 and BG13, that were used in our studies.

Incompressible model: The PP98 law is written in terms of the Elsässer variables $\mathbf{z}^{ \pm}=\mathbf{v} \pm \mathbf{v}_{\mathbf{A}}$, where $\mathbf{v}$ is the plasma flow velocity, $\mathbf{v}_{\mathbf{A}} \equiv \mathbf{B} / \sqrt{\mu_{0} \rho_{0}}$ is the magnetic field normalized to a velocity and $\rho_{0}=\langle\rho\rangle$ is the mean plasma density. It reads in the isotropic case

$$
-\frac{4}{3} \varepsilon_{I} \ell=\left\langle\frac{\left(\delta \mathbf{z}^{+}\right)^{2}}{2} \delta z_{\ell}^{-}+\frac{\left(\delta \mathbf{z}^{-}\right)^{2}}{2} \delta z_{\ell}^{+}\right\rangle \rho_{0} \equiv \mathscr{F}_{I}(\ell),
$$

where the general definition of an increment of a variable $\psi$ is used, i.e. $\delta \psi \equiv \psi(\mathbf{x}+$ $\boldsymbol{\ell})-\psi(\mathbf{x})$. The longitudinal components are denoted by the index $\ell$ with $\ell \equiv|\ell|,\langle\cdot\rangle$ stands for the statistical average and $\varepsilon_{I}$ is the dissipation rate of the total energy. Note that in S.I. units, we have the relation $\rho_{0}=1.673 \times 10^{-21}\left\langle n_{p}\right\rangle$. 
Compressible model: Following the approach used in [Banerjee et al.(2016)], the original equations of the BG13 model can be reduced to the following compact form in the isotropic case

$$
-\frac{4}{3} \varepsilon_{C} \ell=\mathscr{F}_{C+\Phi}(\ell)
$$

where

$$
\mathscr{F}_{C+\Phi}(\ell)=\mathscr{F}_{1}(\ell)+\mathscr{F}_{2}(\ell)+\mathscr{F}_{3}(\ell),
$$

and

$$
\begin{aligned}
& \mathscr{F}_{1}(\ell)=\left\langle\frac{1}{2}\left[\delta\left(\rho \mathbf{z}^{-}\right) \cdot \delta \mathbf{z}^{-}\right] \delta z_{\ell}^{+}+\frac{1}{2}\left[\delta\left(\rho \mathbf{z}^{+}\right) \cdot \delta \mathbf{z}^{+}\right] \delta z_{\ell}^{-}\right\rangle, \\
& \mathscr{F}_{2}(\ell)=\left\langle 2 \delta \rho \delta e \delta v_{\ell}\right\rangle, \\
& \mathscr{F}_{3}(\ell)=\left\langle 2 \bar{\delta}\left[\left(1+\frac{1}{\beta}\right) e+\frac{v_{A}^{2}}{2}\right] \delta\left(\rho_{1} v_{\ell}\right)\right\rangle .
\end{aligned}
$$

where by definition $\bar{\delta} \psi \equiv(\psi(\mathbf{x}+\boldsymbol{\ell})+\psi(\mathbf{x})) / 2, e=c_{s}^{2} \ln \left(\rho / \rho_{0}\right)$ is the internal energy, with $c_{s}$ the constant isothermal sound speed, $\rho$ the local plasma density ( $\left.\rho=\rho_{0}+\rho_{1}\right)$ and $\beta=2 c_{s}^{2} / v_{A}^{2}$ is the local ratio of the total thermal to magnetic pressure $\left(\beta=\beta_{e}+\beta_{p}\right)$. To obtain Equations $(4)-(6)$ several assumptions have been used. These are the assumptions generally adopted in fully developed turbulence theories: infinite kinetic and magnetic Reynolds numbers and a steady state with abalance between forcing and dissipation, statistical homogeneity and stationarity of the turbulent fluctuations [Galtier \& Banerjee(2011) Banerjee \& Galtier(2013)], along with the statistical isotropy. Furthermore, the source terms have been neglected considering that they cannot be estimated using single spacecraft data because of the local spatial divergence involved in those terms, and on the argument that they were found to be sub-dominant (compared to the flux terms) in simulations of supersonic fluid [Kritsuk et al.(2013)] and sub-sonic (isothermal) MHD turbulence [Andrés et al.(2018b)]. For the purpose of applying the model to spacecraft data, the plasma $\beta$ is further assumed to be nearly stationary, which is a stringent requirement in selecting the data used in this work (see details in [Banerjee \& Galtier(2013)] $)$. Note that some of the adopted hypothesis (e.g., homegeneity, isotropy) would be violated because of the expanding nature of the SW [Narita et al.(2010a). Verdini\& Grappin(2016)] or of the presence large scale shears, which then would require adapting the BG13 as done for the case of PP98 model [Wan et al.(2010), Gogoberidze et al.(2013), Hellinger et al.(2013)]. Below, equations (4)- 6) were evaluated using spacecraft data in the fast and slow SW and in the magnetosheath.

\subsection{Application to the slow and fast solar wind}

The two models described above were applied to Cluster and Themis spacecraft observations in the slow and fast wind in order to emphasize the role of density fluctuations. Many aspects were studied and discussed in [Banerjee et al.(2016), Hadid et al.(2017)]. Here, we focus on the role of density fluctuations in enhancing the energy cascade rate (w.r.t. to the incompressible model PP98) and its spatial anisotroy. 
Figure 8 shows a comparison of the ratio between the compressible to the incompressible cascade rate $R=\left\langle\left|\varepsilon_{C}\right|\right\rangle /\left\langle\left|\varepsilon_{I}\right|\right\rangle$ in the fast and slow winds obtained from all the analyzed sample. Here, we use the average value of the cascade rate over all the time lags $\tau$ within the range $10-1000 \mathrm{~s}$ (and not at a single value of $\tau$ as done in previous studies [Stawarz et al.(2009), Coburn et al.(2014)]). This motivated a new criterion applied while selecting our data sample: we kept only intervals of time during which the compressible cascade rate showed a constant (negative or positive) sign for all time lags in the range $10-1000 \mathrm{~s}$.

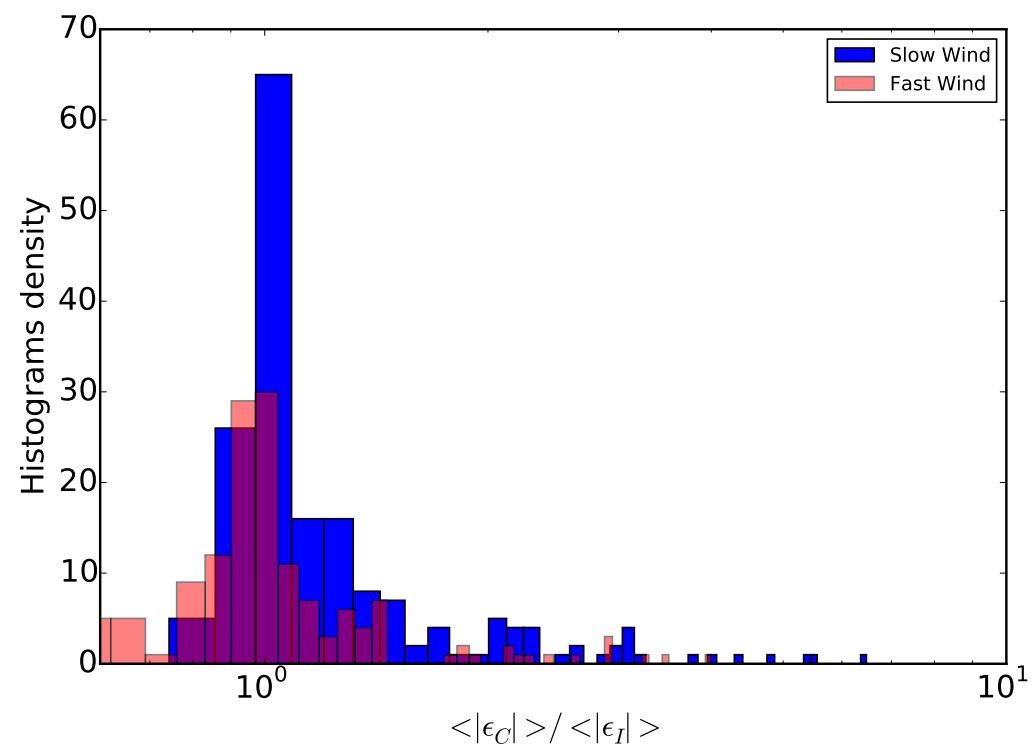

Fig. 8 Histograms of the ratio between the compressible to the incompressible cascade rate $R=$ $\left\langle\left|\varepsilon_{C}\right|\right\rangle /\left\langle\left|\varepsilon_{I}\right|\right\rangle$ in the fast (pink) and slow (blue) winds (adapted from [Hadid et al.(2017)].

A first feature that can be seen in Figure 8 is that the plasma compressibility, while in average may not modify significantly the cascade rate (since the bulk of the distribution of the ratio $R$ is centred around 1), in some cases it does nevertheless amplify it by a factor of $3-4$. This trend is enhanced in the slow wind where the (blue) histogram of $R$ in Figure 8 is found to shift to higher values (up to $7-8$ ) and for a larger number of events than in the fast wind. Note however that these amplification values remain smaller than those reported in [Carbone et al.(2009)] (see the explanation given in [Hadid et al.(2017)]).

To evidence the role of the density fluctuations $\sqrt{\left(\left\langle\rho^{2}\right\rangle-\langle\rho\rangle^{2}\right)} /\langle\rho\rangle$ in enhancing the cascade rate $\left\langle\left|\varepsilon_{C}\right|\right\rangle$ w.r.t. the incompressible one $\left\langle\left|\varepsilon_{I}\right|\right\rangle$ we plotted in Figure 9 $\left\langle\left|\varepsilon_{C}\right|\right\rangle$ as function of the wind speed and the density fluctuations. First, one can see that, overall, the fast wind has a higher $\left\langle\left|\varepsilon_{C}\right|\right\rangle$ than the slow wind. Moreover, one can notice an increase in the cascade rate as compressibility increases in particular in the 


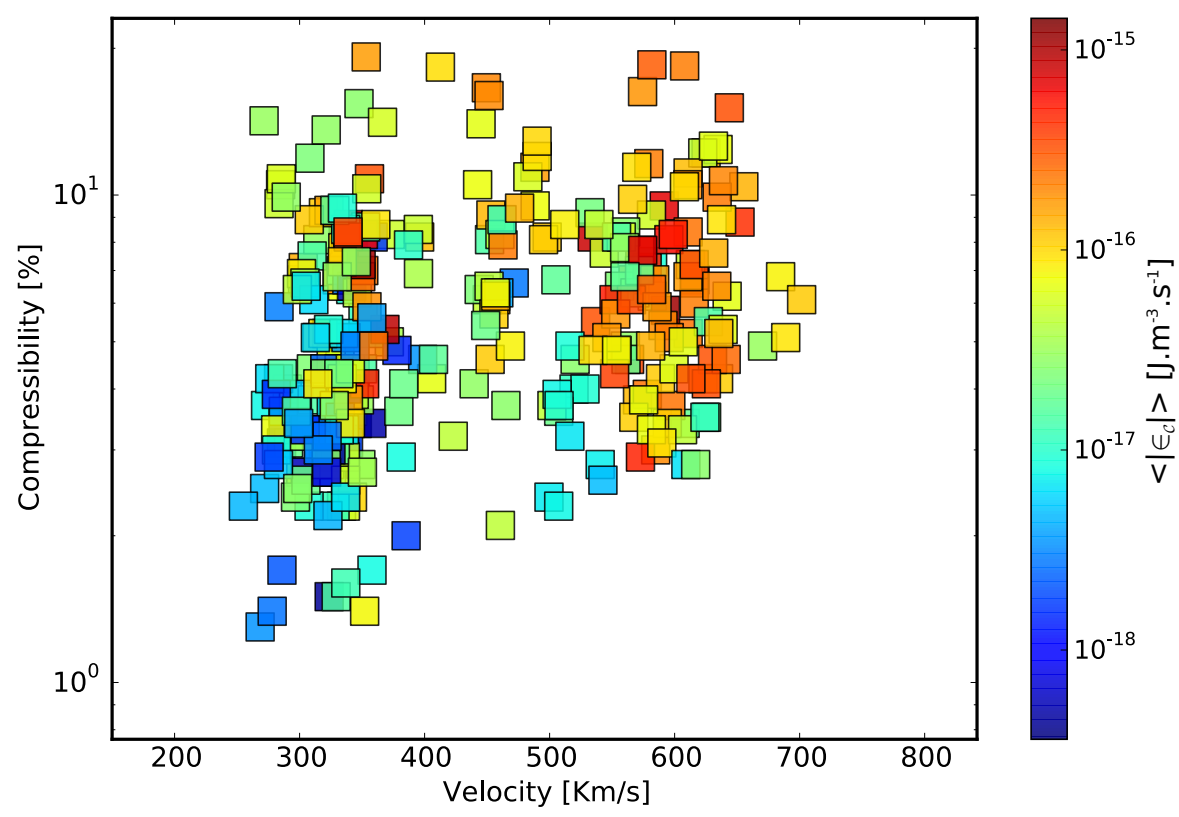

Fig. 9 Variation of the compressible cascade rate $\left\langle\left|\varepsilon_{C}\right|\right\rangle$ as function of the density fluctuations $\delta \rho /\langle\rho\rangle$ (see text) and the wind speed. The vertical color bar gives the magnitude of the cascade rate (adapted from [Hadid et al.(2017)]).

case of the slow wind. This trend is less evident in the case of the fast SW possibly because the spread in the compressibility values is smaller $(\sim 3 \%-15 \%)$ than in the case of the slow wind $(\sim 1 \%-20 \%)$.

Another property that can be explored using in-situ data is the spatial anisotropy of the cascade rate. The anisotropy of the cascade rate has been previously explored using the PP98 model, and it has been shown that the cascade rate is more anisotropic in the fast than in the slow SW [MacBride, Smith \& Forman(2008)]. In the previous works, the original PP98 equations were modified to fit the limit of either 1D (slab) and 2D geometry, through the appropriate projection of the flux terms onto the two directions parallel and perpendicular to the mean magnetic field. Here, we do not use that approach for either the PP98 or the BG13 models. Instead, we simply examine the dependence of the estimated cascade rates on the angle $\Theta_{\mathbf{V B}}$. As we explained above, the use of the Taylor hypothesis $(l=-V \tau)$ to convert time lags $\tau$ into spatial scales implies that the analysis samples only the direction along the SW flow. Thus, when $\Theta_{\mathbf{V B}} \sim 0^{\circ}$ (resp. $\Theta_{\mathbf{V B}} \sim 90^{\circ}$ ) the analysis yields information in the direction parallel (resp. perpendicular) to the local mean magnetic field. Therefore, estimating the cascade rate as function of the sampling direction of space given by the angle $\Theta_{\mathrm{VB}}$ should allow us to gain insight into the anisotropic nature of the cascade. We used this approach by spliting our statistical samples (in the fast and slow winds) as function of the angle $\Theta_{\mathbf{V B}}$. The result is given in Figure 10. Two important observations 




Fig. 10 Estimated energy cascade rates from BG13 and PP98 as a function of the angle $\Theta_{\mathbf{V B}}$ and the total compressible energy $E_{1}^{\text {comp }}$ in the fast (Left) and slow (Right) solar wind. The blue curve represents the ratio $R=\left\langle\left|\varepsilon_{C}\right|\right\rangle /\left\langle\left|\varepsilon_{I}\right|\right\rangle$ as a function of $\Theta_{\mathbf{V B}}$ (adapted from [Hadid et al.(2017)]).

can be made. Both models, PP98 and BG13, provide a cascade rate that is strongly depending on the angle $\Theta_{\mathbf{V B}}$ (as we will see below, this result contrasts with the one obtained in the magnetosheath that has higher compressibility, see section 5.3 . The dependence on the angle $\Theta_{\mathbf{V B}}$ is even more pronounced in the slow wind than in the fast wind. This contrasts with the finding of [MacBride, Smith \& Forman(2008)] who showed no significant anisotropic cascade in the slow wind. The reason of this discrepancy may come from the criterion of uniform angle $\Theta_{\mathrm{VB}}$ used in this work, which allows us to better evidence the difference in the cascade rates parallel and perpendicularly to the mean field.

\subsection{Application to the terrestrial magnetosheath}

As explained in section 4 , the magnetosheath plasma shows in general large density fluctuations, which makes the use of the BG13 model necessary if one wants to estimate the energy cascade rate in the medium, although this model is derived within the isothermal assumption whose application to the magnetosheath and SW plasmas can be questioned. This was first done in [Hadid et al.(2018)]. Here, we recall some of their major findings.

A similar procedure of data selection as in [Banerjee et al.(2016) Hadid et al.(2017)] was adopted in this study. Furthermore, only intervals of time that showed Kolmogorovlike spectrum $\left(\sim f^{-5 / 3}\right)$ were retained. To further highlight the role of density fluctuations, the data intervals were split into two sub-sets: one dominated by incompressible Alfvénic fluctuations and the other by compressible magnetosonic-like fluctuations using the magnetic compressibility criterion as explained in section 4.1

For each of these groups we computed the absolute values of the cascade rates $\left|\varepsilon_{C}\right|$ and $\left|\varepsilon_{I}\right|$ from the compressible BG13 and the incompressible model PP98, respectively. In this study we considered the magnitude of the cascade rate rather than its signed value (assuming that the former is statistically representative of the actual 

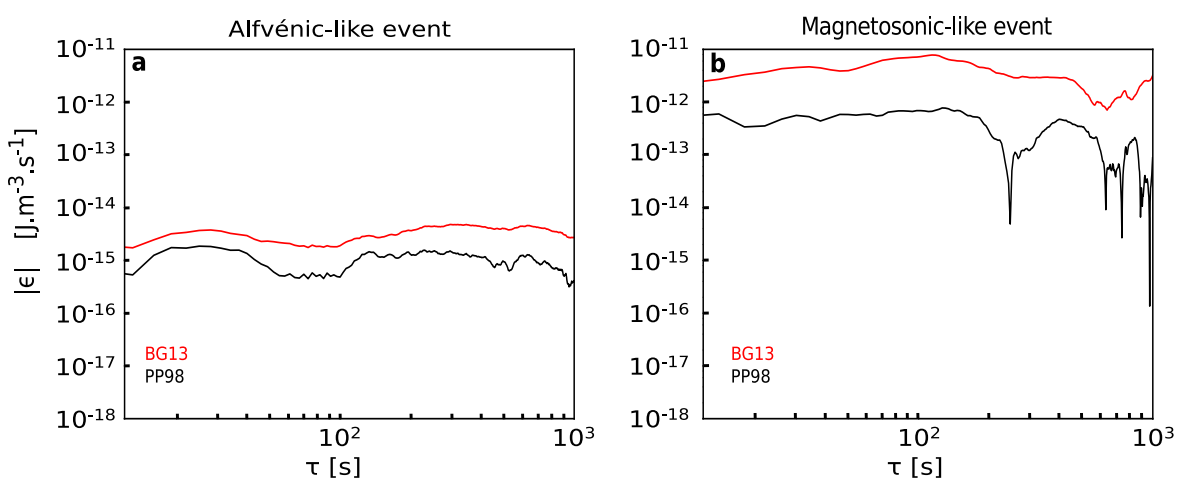

Fig. 11 The energy cascade rates computed using BG13 (red) and PP98 (black) for (a) an Alfvénic turbulence and (b) magnetosonic-like one (adapted from [Hadid et al.(2018)]).

cascade rate). This is because signed cascade rates require very large statistical samples to converge [Coburn et al.(2015), Hadid et al.(2017)], which are not available to us for this study. However, by applying a linear fit on the resulting energy cascade rates, we considered only the ones that are relatively linear with $\tau$ and showed no sign change at least over one decade of scales in the inertial range. Two main observations can be made from the two examples shown in Figure 11, first, the incompressible cascade rate $\left|\varepsilon_{I}\right|$ is larger by a factor $\sim 100$ in the magnetosonic case compared to the Alfvénic one, which can be explained by the large amplitude $\delta B$ in the former [Hadid et al.(2017)]. Second, density fluctuations in the magnetosonic case amplify $\left|\varepsilon_{C}\right|$ by a factor $\sim 7$ w.r.t. $\left|\varepsilon_{I}\right|$. Overall, it was found that for the incompressible Alfvénic cases, the values of $\left\langle\left|\varepsilon_{C}\right|\right\rangle$ and $\left\langle\left|\varepsilon_{I}\right|\right\rangle$ are very similar with a mean $\sim 10^{-14} J . m^{-3} \cdot s^{-1}$, whereas for the compressible magnetosonic events the values of $\left\langle\left|\varepsilon_{C}\right|\right\rangle$ are higher than those of $\left\langle\left|\varepsilon_{I}\right|\right\rangle$. The corresponding mean values are respectively $\sim 6 \times 10^{-13}$ and $\sim 2 \times 10^{-13} \mathrm{~J} . \mathrm{m}^{-3} . \mathrm{s}^{-1}$.

Interestingly, the role of the compressibility in increasing the compressible cascade rate can be evidenced by the turbulent Mach number $\mathscr{M}_{s}=\sqrt{\langle\delta v\rangle^{2} / c_{s}^{2}}$, where $\delta v$ is the fluctuating flow velocity. Figure 12 shows a power law-like dependence of $\left\langle\left|\varepsilon_{C}\right|\right\rangle$ on $\mathscr{M}_{s}$ as $\left\langle\left|\varepsilon_{C}\right|\right\rangle \sim \mathscr{M}_{s}^{4}$, steeper than the one observed in the SW [Hadid et al.(2017)]. We are not aware of any theoretical prediction that relates $\varepsilon$ to $\mathscr{M}_{s}$ in compressible turbulence. However, in incompressible flows, dimensional analysis à la Kolmogorov yields a scaling that relates $\varepsilon_{I}$ to the third power of $\mathscr{M}_{s}$. The high level of the density fluctuations in the magnetosheath seems to modify this scaling to the one we estimated here. Although more analytical and numerical studies are needed to understand the relationship between $\left|\varepsilon_{C}\right|$ and $\mathscr{M}_{s}$, the scaling law recalled here may be used as an empirical model for other compressible media non accessible to in-situ measurements such as the ISM (if the scaling obtained here proves to be valid for supersonic turbulence).

The spatial anisotropy of the cascade rate is explored via the dependence of the estimated cascade rates on the mean angle $\Theta_{\mathbf{V B}}$ between the local magnetic and flow vectors, as it has been already done in similar studies of SW turbulence [Smith et al.(2009) Marino et al.(2011) Hadid et al.(2017)]. 


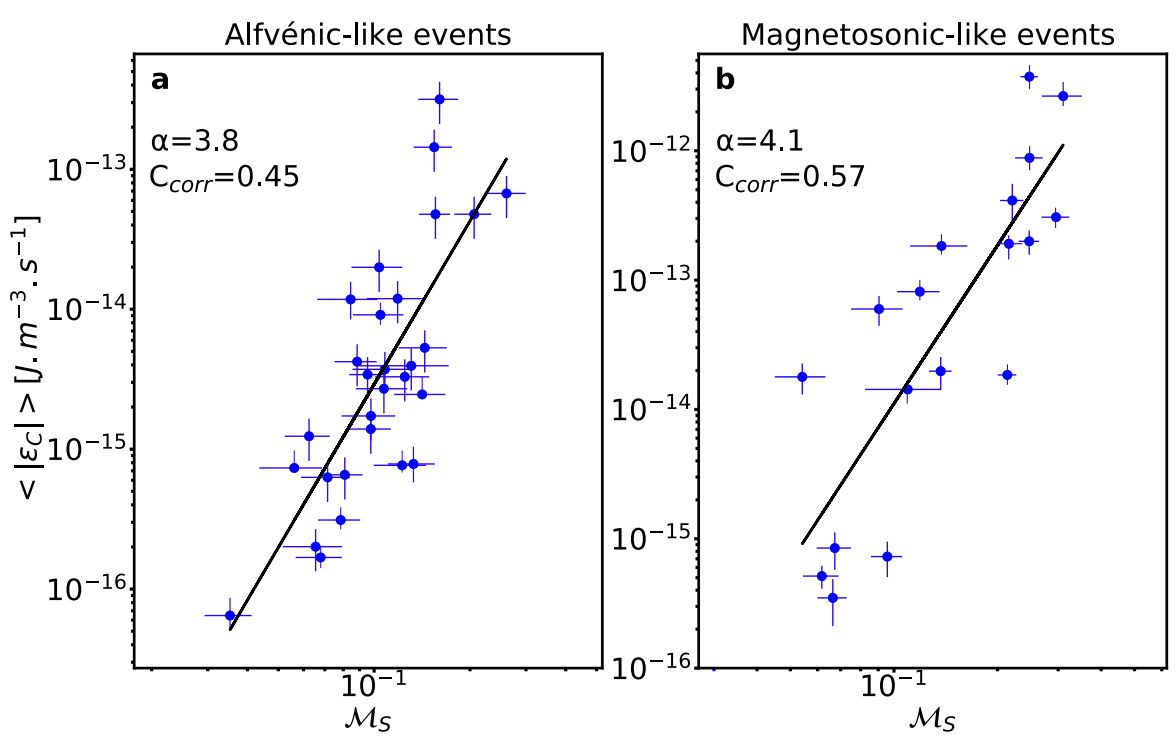

Fig. 12 Compressible energy cascade rate as a function of the turbulent Mach number for the Alfvénic (a) and Magnetosonic-like (b) events. The black line represents a least square fit of the data, $\alpha$ is the slope of the power-law fit. The error bars represent the standard deviation of $\left|\varepsilon_{C}\right|$ (adapted from [Hadid et al.(2018)]).


Fig. 13 Compressible and incompressible energy cascade rates $\left\langle\left|\varepsilon_{C}\right|\right\rangle$ and $\left\langle\left|\varepsilon_{I}\right|\right\rangle$ as a function of the mean angle $\Theta_{\mathbf{V B}}$ and the total energy (colored bar) for the Alfvénic (a) and magnetosonic (b) events. The blue line is the ratio $\mathscr{R}=\left\langle\left|\varepsilon_{C}\right|\right\rangle /\left\langle\left|\varepsilon_{I}\right|\right\rangle$ (adapted from [Hadid et al.(2018)] $)$.

As one can see in Figure 13, for both models the cascade rate is lower in the parallel direction than in the perpendicular one. For the magnetosonic events the highest cascade rate and total energy are observed at oblique angles $\Theta_{\mathbf{V B}} \sim 50^{\circ}-60^{\circ}$. The second observation is that the density fluctuations seem to reinforce the anisotropy of the cascade rate w.r.t. the Alfvénic turbulence: The ratio $\mathscr{R}=\left\langle\left|\varepsilon_{\mathscr{C}}\right|\right\rangle /\left\langle\left|\varepsilon_{\mathscr{I}}\right|\right\rangle$ (in blue) is close to 1 for the Alfvénic cases, but increases to $\sim 3$ for the magnetosonic ones at quasi-perpendicular angles. Numerical simulations of compressible MHD tur- 
bulence showed that fast magnetosonic turbulence is spatially isotropic while slow mode turbulence is anisotropic and has a spectrum $k_{\perp}{ }^{-5 / 3}$ similarly to Alfvénic turbulence [Cho \& Lazarian(2002)]. This first observation that density fluctuations enhance the anisotropy of the cascade rate suggests that a slow-like (or mirror) mode turbulence dominates the compressible fluctuations analyzed here [Sahraoui et al.(2006). Hadid et al.(2015)]. This result agrees with the analysis of the stability conditions of
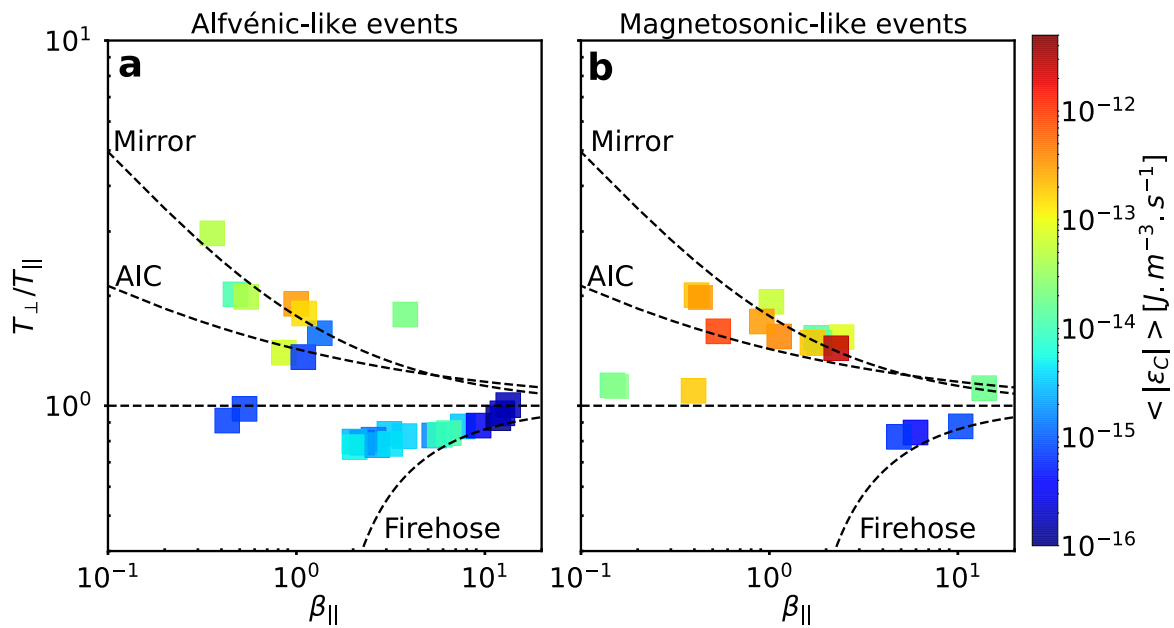

Fig. 14 Compressible energy cascade rate $\left\langle\left|\varepsilon_{C}\right|\right\rangle$ averaged into bins of proton temperature anisotropy $\left(T_{\perp} / T_{\|}\right)$vs $\beta_{\|}$for (a) the Alfvénic and (b) magnetosonic-like events. The dashed lines correspond to the mirror, the AIC, and firehose linear instabilities thresholds (adapted from Hadid et al.(2018)]).

the plasma derived from the linear Maxwell-Vlasov theory. Figure 14 shows that a large fraction of the lowest values of the compressible cascade rate corresponds to alfvénic-like events and lie close to the stability condition $T_{\perp} / T_{\|} \sim 1$. In contrast, most of the highest compressible cascade rates correspond to magnetosonic events and lie near the mirror more instability threshold. Considering that the maximum growth rate of the linear mirror instability occurs at oblique angles $\Theta_{\mathbf{k B}}$ (approximated here by the angle $\Theta_{\mathbf{V B}}$ ) [Pokhotelov et al.(2004)], the peak of the cascade rate observed for $\Theta_{\mathbf{V B}} \sim 50^{\circ}-60^{\circ}$ in Fig. 13 may be explained by energy injection into the background turbulent plasma through the mirror instability, which seems to enhance the cascade rate. A similar relationship was found in the SW between the incompressible cascade rate and kinetic plasma instabilities [Osman et al.(2013)], however, deeper understanding of the connection between these two features of plasma turbulence requires further theoretical investigation [Kunz et al.(2014)].

It is important to emphasize here that while the MHD models we used above allow us to obtain an estimation of the energy cascade rate, they do not tell us how this energy is eventually dissipated at the kinetic scales. This fundamental aspect will not be discussed here, although it is clearly a subject of heated debates within the community because several (sometimes competing) dissipation mechanisms are proposed. These include wave-particles interaction (e.g., Landau, cyclotron) [Landau(1946) 
Howes et al.(2008) Schekochihin et al.(2009) Sahraoui et al.(2010a) Sulem \& Passot(2015), Cranmer(2014), He et al.(2015), Kobayashi et al.(2017)], stochastic heating [Chandran et al.(2010)], or dissipation by magnetic reconnection within localized structures [Sundvist et al.(2007), Retino et al.(2007) Servidio et al.(20111), Chasapis et al.(2015)]. An intensive research work is currently being done to identify the signature in the particle VDFs associated to each dissipation mechanism, both in numerical simulations (GK or Vlasov) and MMS data [Howes et al.(2017), Klein et al.(2017), Chen et al.(2019)], and to investigate the process of cascade in velocity space via Landau damping and its possible cancellation by the stochastic plasma echo [Schekochihin et al.(2016), Servidio et al.(2017), Cerri et al.(2018), Meyrand et al.(2019)].

\section{Other features of magnetosheath turbulence: coherent structures}

Another important feature of the plasma turbulence that is subject to deep investigation is the nature of the coherent structures that form in turbulent plasmas and their role in particle energization [Matthaeus et al.(1984) Servidio et al.(20111) Loureiro et al.(2013) Karimabadi et al.(2013)]. Indeed, a nonlinear energy cascade in magnetized turbulent plasmas generates self-consistently different coherent structures such as mirror modes [Horbury et al.(2004), Sahraoui et al.(2008)], magnetic islands/flux ropes [Daughton et al.(2011),Karimabadi et al.(2014), Huang et al.(2016)], Alfvén vortices [Alexandrova et al.(2006)], current sheets and other discontinuities [Retino et al.(2007), Servidio et al.(20111) Osman et al.(2012), Chasapis et al.(2015)], which all were reported in the magnetosheath. In turbulence jargon these structures are generally referred to as intermittency.

Among the myriad of coherent structures that can be generated in magnetized turbulent plasmas, current sheets are certainly the ones that attract more attention in turbulence studies because of the important role they play in dissipating energy via magnetic reconnection [Matthaeus et al.(1984), Sundvist et al.(2007), Servidio et al.(2014)]. Figure 16 shows an example of energy "dissipation" (i.e., a sudden increase of J.E) in a thin current sheet observed in Cluster data in the magnetosheath. More recent statistical studies have evidenced the role of thin (proton-scale) current sheets in heating electrons in the turbulent magnetoseath plasma [Chasapis et al.(2015)].

Addressing the vast topic of coherent structures and their role in local particle energization is beyond the scope of this paper. In this section we only give a small glimpse of the new small scale structures evidenced recently by MMS in the magnetosheath. Indeed, thanks its unprecedented time resolution (in particular that of the plasma instruments [Burch et al.(2016), Pollock et al.(2016)]), MMS data allows us to probe into nearly electron-scale plasma dynamics, where new types of coherent structures were evidenced along with their role energizing locally the plasma particles. This is the case of sub-ion scale magnetic holes/dips, found to be associated to intense wave activity and electron dynamics [Gershman et al.(2016), Yao et al.(2017), Yao et al.(2019)] and ion-scale magnetic islands [Huang et al.(2016)], which had been reported earlier in global hybrid [Karimabadi et al.(2014)] and PIC [Roytershteyn et al.(2015)] simulations. In the latter, MMS data showed intense particle dynamics and wave ac- 


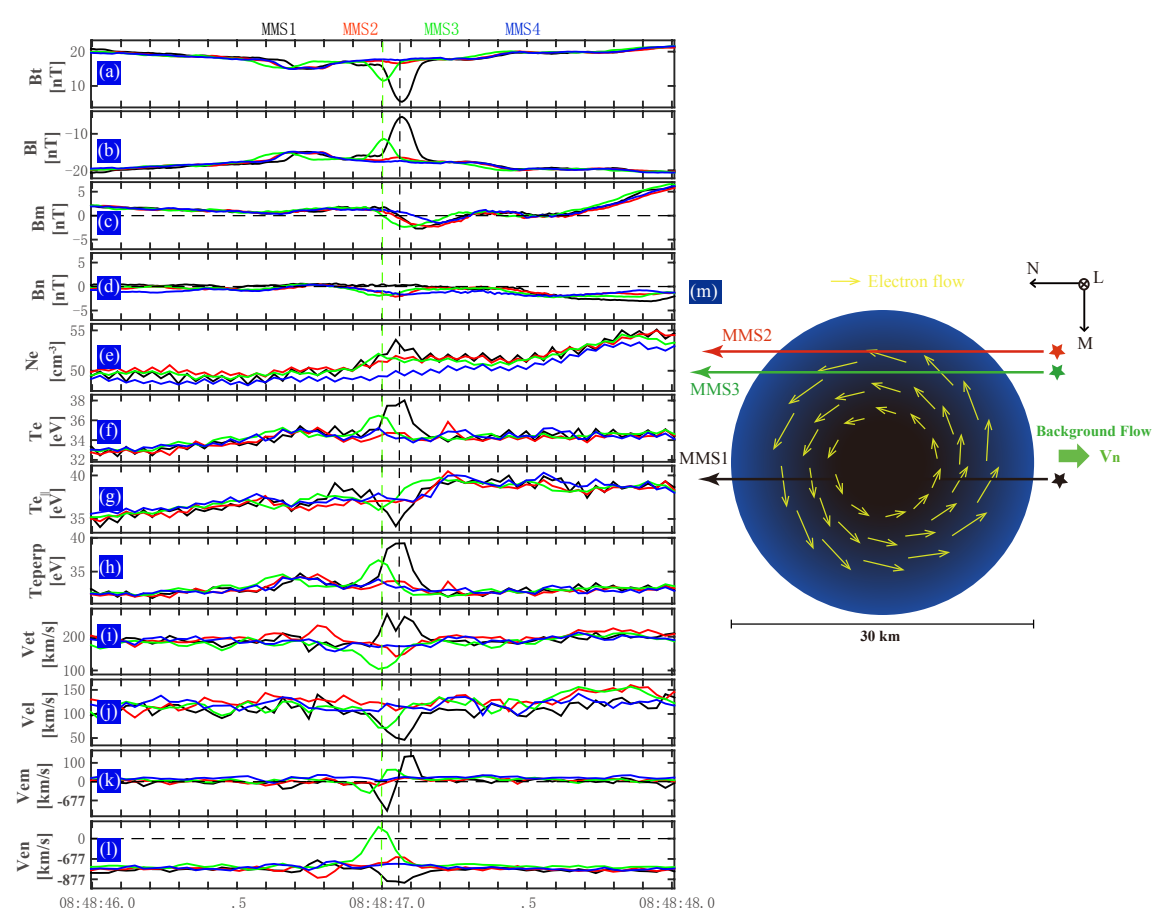

Fig. 15 Detailed observations of the electron vortex magnetic hole in the magnetosheath, and schematic of the electron vortex magnetic hole in the MN plane (adapted from Huang et al. 2017a).

tivity (e.g., electron beams, electrostatic solitary structures, strong electromagnetic lower hybrid drift waves) [Huang et al.(2016)].

At even smaller scales, MMS data provided evidence of a new type of electronscale coherent structure, referred to as electron vortex magnetic holes, in the turbulent magnetosheath plasma [Huang et al.(2017a),Huang et al.(2017b)]. An example is shown in Figure 15. These electron-scale magnetic holes are characterized by magnetic field strength depression, electron density enhancement, significant heating (resp. cooling) in the perpendicular (resp. parallel) directions, and an electron vortex formed by the trapped electrons [Huang et al.(2017a)]. The strong increase of electron temperature indicates that these magnetic holes have strong connection with the energization of electrons [Huang et al.(2017b) Huang et al.(2018)]. The wealth of information on small scale structures provided by MMS data calls now for new theoretical (not only numerical) works in order to better understand how those structures form and elucidate their role in energizing the plasma particles.

\section{Conclusions and some perspectives}

The selection of results presented in this paper provides only a glimpse of the various facets of nonlinear dynamics in turbulent magnetized plasmas. They emphasize some 


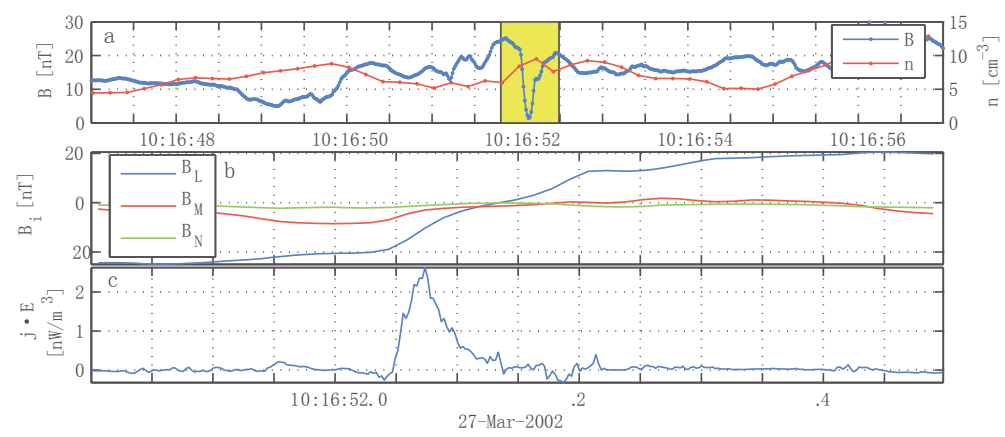

Fig. 16 Observation of thin current sheet in the magnetosheath (after Sundkvist et al. 2007). (a) Magnetic field and density fluctuations. The yellow patch is shown in detail in panels the panels below. (b) Reconnecting component of magnetic field (BL), out-of- plane component (BM), normal component (BN). (c) Dissipated power per unit volume.

of the questions that in-situ measurements taken in different regions of the near-Earth space can help to resolve. The data analysis is supported by the state of the art numerical simulations and theories developed within different plasma approximations (MHD, Hall-MHD, Landau-fluid, PIC, hybrid, GK and Vlaosov codes).

A clear trend toward investigating kinetic scale physics emerges from these studies. These ongoing efforts target the identification of the nature of the turbulent fluctuations at the sub-ion and electron scale, the actual mechanisms of energy dissipation and its consequences in terms of particle energization, and the interplay between waves and coherent structures. While space missions such as MMS, PSP and Solar Orbiter (scheduled for launch in 2020) already (or will soon) provide crucial information on the dynamics of the kinetic scales, a need for a new mission that is fully dedicated to the very small (electron scale) physics emerged in recent years. The new mission is expected to have higher sensitivity electric and magnetic field measurements (Fig. 17) to capture small amplitude fluctuations at electron scales in the SW at $1 \mathrm{AU}$ and high time, energy and angular resolution of the plasma particles (ions and electrons) to allow for measuring a well-resolved velocity space, where key signatures of energy dissipation can be detected [Grison et al.(2005), He et al.(2015), Schekochihin et al.(2016), Howes et al.(2017) Servidio et al.(2017) Cerri et al.(2018), Chen et al.(2019), Meyrand et al.(2019)]. These needs were reflected in recent years by proposing new missions to tackle these science questions such us THOR (candidate to the ESA M4 call, 2015-2017, [Vaivads et al.(2016)]) and Debye (candidate to the ESA F1 call, 2018-2019, [Verscharen et al.(2019)]). Although these missions were not selected, they nevertheless paved the road for a future multi-spacecraft mission that would solve the problem of the energy dissipation at small (electron) scales in the SW. 


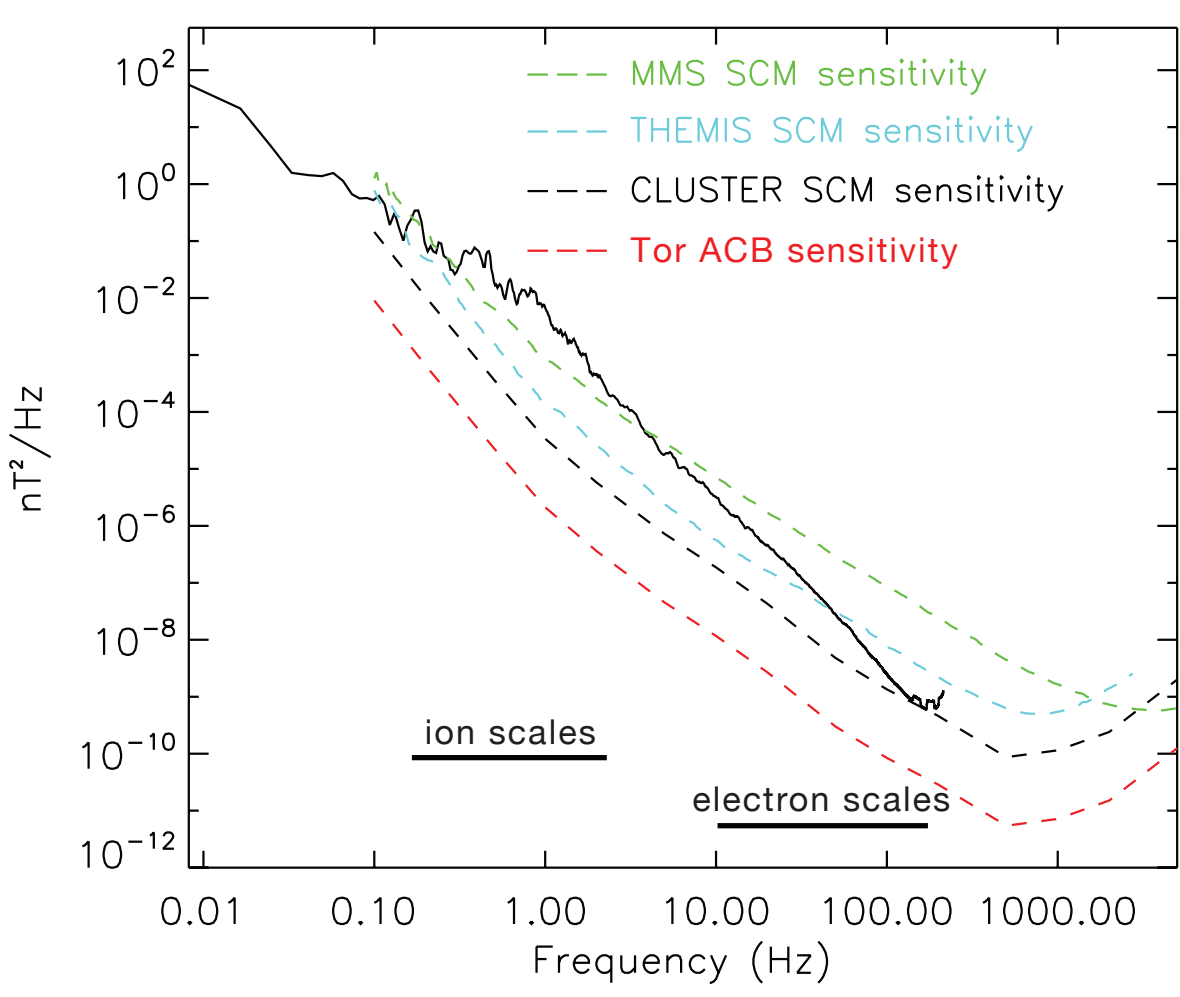

Fig. 17 The sensitivity of the search-coil magnetometer targeted by mission proposals dedicated to the study of electron scale turbulence in the solar wind such as THOR or Debye (red), compared to the sensitivity of similar instruments that flew on previous spacecraft. The solid line shows a turbulence spectrum in solar wind, measured by Cluster, when the turbulence amplitude is high.

\section{Acknowledgements}

\section{References}

Alexandrova et al.(2006). Alexandrova, O., A. Mangeney, M. Maksimovic, N. Cornilleau-Wehrlin, J.-M. Bosqued, and M. André, Alfvén vortex filaments observed in magnetosheath downstream of a quasi-perpendicular bow shock, J. Geophys. Res., 111, A12208 (2006)

Alexandrova et al.(2008). Alexandrova, O., C Lacombe, \& A Mangeney, Spectra and anisotropy of magnetic fluctuations in the earth's magnetosheath: Cluster observations, Ann. Geophys., 26:3585 (2008)

Alexandrova et al.(2012). Alexandrova, O., C. Lacombe, A. Mangeney, R. Grappin, \& M. Maksimovic, SOLAR WIND TURBULENT SPECTRUM AT PLASMA KINETIC SCALES, The Astrophysical Journal, 760:121 (2012)

Andrés et al.(2016). Andrés, N., S. Galtier, \& F. Sahraoui, Exact scaling laws for helical threedimensional two-fluid turbulent plasmas, Phys. Rev. E 94, 063206 (2016)

Andrés \& Sahraoui(2017). Andrés, N. \& F. Sahraoui, Alternative derivation of exact law for compressible and isothermal magnetohydrodynamics turbulence, Phys. Rev. E 96, 053205 (2017)

Andrés et al.(2018a). Andrés, N., S. Galtier, \& F. Sahraoui, Exact law for homogeneous compressible Hall magnetohydrodynamics turbulence, Phys. Rev. E 97, 013204 (2018) 
Andrés et al.(2018b). Andrés, N., S. Galtier, F. Sahraoui, L. Z. Hadid, P. Dmitruk, \& P. D. Mininni, Energy cascade rate in isothermal compressible magnetohydrodynamic turbulence, J. Plasmas Phys. 84 , $905840404(2018)$

Balbus et al.(1998). S. A. Balbus and J. F. Hawley. Reviews of Modern Physics, 70, 1998

Bale et al.(2005). Bale, S. D. , Kellogg, P. J. , Mozer, F. S. , Horbury, T. S. , \& Rème, H. 2005, Phys. Rev. Lett., 94, 215002

Banerjee \& Galtier(2013). Banerjee, S., \& Galtier, S. 2013, Phys. Rev. E, 87, 013019

Banerjee et al.(2016). Banerjee, S., Hadid, L.Z., Sahraoui, F. \& Galtier, S., SCALING OF COMPRESSIBLE MAGNETOHYDRODYNAMIC TURBULENCE IN THE FAST SOLAR WIND, The Astrophysical Journal Letters, 829:L27 (2016)

Banerjee \& Galtier(2017). Banerjee, S., \& S. Galtier, J. Phys. A: Math. Theo. 50,015501 (2017)

Brandenburg et al.(2011). Brandenburg A and Nordlund E. arxiv:0912.1340v2. The Astrophysical Journal Supp., 2011

Belmont \& Rezeau(2001). Belmont G. \& L. Rezeau, J. Geophys. Res., 106 (2001)

Boldyrev \& Perez(2012). Boldyrev, S. and J. C. Perez, SPECTRUM OF KINETIC-ALFVEN TURBULENCE, ApJL, 758:L44 (2012)

Boldyrev et al.(2013). Boldyrev, S., K. Horaites, Q. Xia, and J. C. Perez, TOWARD A THEORY OF ASTROPHYSICAL PLASMA TURBULENCE AT SUBPROTON SCALES, ApJ, 777:41 (2013)

Bourouaine \& Perez(2018). Bourouaine, S. and J. C. Perez, On the Limitations of Taylors Hypothesis in Parker Solar Probes Measurements near the Alfvn Critical Point, ApJL, 858:L20 (2018)

Bruno \& Carbone(2005). Bruno, R., \& Carbone, V. 2005, Living Rev. Solar Phys., 2, 4

Bruno et al.(2014). Bruno, L. Trenchi, \& D. Telloni, SPECTRAL SLOPE VARIATION AT PROTON SCALES FROM FAST TO SLOW SOLAR WIND, The Astrophysical Journal Letters, 793:L15 (2014)

Burch et al.(2016). Burch, J. L., T. E. Moore, R. B. Torbert, and B. L. Giles, Magnetospheric Multiscale overview and science objectives, Space Sci. Rev., doi:10.1007/s11214-015-0164-9 (2016)

Carbone et al.(2009). Carbone, V., Marino, R., Sorriso-Valvo, L., et al. 2009, Phys. Rev. Lett., 103, 061102

Chandran et al.(2010). Chandran, B. D. G. , B. Li, B. N. Rogers, E. Quataert, \& K. Germaschewski, Perpendicular Ion Heating by Low-frequency Alfven-wave Turbulence in the Solar Wind, Astrophys. J. , 720:503515 (2010)

Chandran (2018). Chandran, B. D. G., Parametric instability, inverse cascade and the $1 / \mathrm{f}$ range of solarwind turbulence, J. Plasma Phys., vol. 84, 905840106 (2018)

Chasapis et al.(2015). Chasapis, A., A. Retino, F. Sahraoui, A. Vaivads, Yu. V. Khotyaintsev, D. Sundkvist, A. Greco, L. Sorriso-Valvo, \& P. Canu, THIN CURRENT SHEETS AND ASSOCIATED ELECTRON HEATING IN TURBULENT SPACE PLASMA, Astrophys. J. Lett., 804:L1 (2015)

Coburn et al.(2014). Coburn, J.T., et al. 2014, Astrophys. J., 786, 52

Coburn et al.(2015). Coburn, J.T., et al. 2015, Phil. Trans. R. Soc. A, 373, 20140150

Chen et al.(2017). Chen, C. H. K. \& S. Boldyrev, Nature of Kinetic Scale Turbulence in the Earths Magnetosheath, Astrophys. J., 842:122 (2017)

Chen et al.(2019). Chen, C. H. K. , K.G. Klein, \& G.G. Howes, Evidence for electron Landau damping in space plasma turbulence, Nature Com., 10:740 (2019)

Cerri et al.(2017b). Cerri, S. S., F. Califano, F. Jenko, D. Told, and F. Rincon, SUBPROTON-SCALE CASCADES IN SOLAR WIND TURBULENCE: DRIVEN HYBRID-KINETIC SIMULATIONS, 822:L12 (2016)

Cerri \& Califano(2017). Cerri, S. S. and F. Califano, Reconnection and small-scale fields in 2D-3V hybrid-kinetic driven turbulence simulations, New J. Phys. 19, 025007 (2017)

Cerri et al.(2018). Cerri, S.S., M. W. Kunz, and F. Califano, Dual Phase-space Cascades in 3D HybridVlasovMaxwell Turbulence, The Astrophysical Journal Letters, 856:L13 (2018)

Cho \& Lazarian(2002). Cho, J., \& A. Lazarian, Compressible Sub-Alfvenic MHD Turbulence in LowPlasmas, Phys. Rev. Lett. 88, 245001 (2002)

Coleman(1968). Coleman, Jr., P. J., 1968, ApJ, 153:371

Cranmer(2014). , Cranmer, S. R., Ensemble simulations of proton heating in the solar wind via turbulence and ion cyclotron resonance, ApJ Supp. Series, 213:16 (2014)

Daughton et al.(2011). Daughton, W., V. Roytershteyn, H. Karimabadi, L. Yin, B. J. Albright, B. Bergen and K. J. Bowers, Role of electron physics in the development of turbulent magnetic reconnection in collisionless plasmas, Nature Physics volume 7, pages539542 (2011)

Diamond et al.(2005). Diamond P. H., S-I Itoh, K Itoh, and T S Hahm. Plasma Phys. Control. Fusion, 47:R35, 2005 
Dmitruk et al.(2007). Dmitruk, P., \& Matthaeus, W. H., Phys. Rev. E 76, 036305 (2007)

Dmitruk et al.(2011). Dmitruk, P., P. D. Mininni, \& A. Pouquet, Phys. Rev.E 83, 066318 (2011)

Federrath et al.(2016). Federrath, C., Magnetic field amplification in turbulent astrophysical plasmas, J. Plasma Phys. (2016), 82, 535820601 (2016)

Ferrand et al.(2019). Ferrand, R., S. Galtier, F. Sahraoui, R. Meyrand, N. Andrés, S. Banerjee, On exact laws in incompressible Hall magnetohydrodynamic turbulence, Astrophy. J. (in press)

Fox et al.(2016). Fox, N. J., M. C. Velli, S. D. Bale, R. Decker, A. Driesman, R. A. Howard, J. C. Kasper, J. Kinnison, M. Kusterer, D. Lario, M. K. Lockwood, D. J. McComas, N. E. Raouafi, and A. Szabo. The Solar Probe Plus Mission: Humanitys First Visit to Our Star. Space Sci. Rev., 204:748 (2016)

Frisch(1995). Frisch, U. 1995, Turbulence; the legacy of A.N. Kolmogorov (Cambridge: Cambridge Univ. Press)

Franci et al.(2017). Franci, L., S. S. Cerri, F. Califano, S. Landi, E. Papini, A. Verdini, L. Matteini, F. Jenko, and P. Hellinger, Magnetic Reconnection as a Driver for a Sub-ion-scale Cascade in Plasma Turbulence, ApJL, 850:L16 (2017)

Galtier (2006).

Galtier (2008). Galtier, S. 2006, Phys. Rev. E 77, 015302

Galtier \& Banerjee(2011). Galtier, S., \& Banerjee, S. 2011, Phys. Rev. Lett., 107, 134501

Gary \& Smith(2009). Gary, S. P. \& Smith, C. W. 2009, J. Geophys. Res., 114, A12105

Gary et al.(2012). Gary, S. P. , O. Chang, and J. Wang, The Astrophysical Journal 755, 142 (2012)

Gershman et al.(2016). Gershman, D. J., J. C. Dorelli, A. F. Vias, L. A. Avanov, U. Gliese, A. C. Barrie, V. Coffey, M. Chandler, C. Dickson, E. A. MacDonald, C. Salo, M. Holland, Y. Saito, J. A. Sauvaud, B. Lavraud, W. R. Paterson, R. Torbert, L. J. Chen, K. Goodrich, C. T. Russell, R. J. Strangeway, B. L. Giles, C. J. Pollock, T. E. Moore, and J. L. Burch, Electron dynamics in a subprotongyroscale magnetic hole, Geophys. Res. Lett., 43, 41124118, doi:10.1002/2016GL068545 (2016)

Gogoberidze et al.(2013). Gogoberidze, G., S. Perri, and V. Carbone, THE YAGLOM LAW IN THE EXPANDING SOLAR WIND, ApJ 769:111 (2013)

Goldstein et al.(1994). Goldstein, M. L., Roberts, M. D., \& Fitch, C., Properties of the fluctuating magnetic helicity in the inertial and dissipation ranges of solar wind turbulence, J. Geophys. Res., 99, 11519 (1994)

Goldstein et al.(1995). Goldstein, M. L., A. D. Roberts, W. H. Matthaeus, MAGNETOHYDRODYNAMIC TURBULENCE IN THE SOLAR WIND, Annu. Rev. Astron. Astrophys., 33:283-325 (1995)

Grison et al.(2005). Grison, B., F. Sahraoui, B. Lavraud, T. Chust, N. Cornilleau-Wehrlin, H. Rème, A Balogh \& M. André, Wave Particle Interaction in the Distant Cusp Region : a Cluster Case Study, Ann. Geophys. 23, 3699-3713 (2005)

Hadid et al.(2015). Hadid, L.Z., Sahraoui, F., K. H. Kiyani, A. Retino, R. Modolo, P. Canu, A. Masters, \& M. K. Dougherty, NATURE OF THE MHD AND KINETIC SCALE TURBULENCE IN THE MAGNETOSHEATH OF SATURN: CASSINI OBSERVATIONS, The Astrophysical Journal Letters, 813:L29 (2015)

Hadid et al.(2017). Hadid, L.Z., Sahraoui, F. \& Galtier, S., Energy Cascade Rate in Compressible Fast and Slow Solar Wind Turbulence, The Astrophysical Journal, 838:9 (2017)

Hadid et al.(2018). Hadid, L. Z., F. Sahraoui, S. Galtier, S. Y. Huang, Compressible magnetohydrodynamic turbulence in the Earth's magnetosheath: estimation of the energy cascade rate using in situ spacecraft data, Phys. Rev. Lett. 120,055102 (2018)

He et al.(2011). He, J. S., E. Marsch, C. Y. Tu, Q. G. Zong, S. Yao, \& H. Tian, Journal of Geophysical Research 116, A06207 (2011)

He et al.(2012). He, J. S., C. Tu, E. Marsch, and S. Yao, The Astrophysical Journal Letters 745, L8 (2012)

He et al.(2015). He, J. S., Wang, L., Tu, C., Marsch, E. \& Zong, Q., Evidence of Landau and cyclotron resonance between protons and kinetic waves in solar wind turbulence, Astrophys. J. 800, L31 (2015)

Hellinger et al.(2013). Hellinger, P., P. M. Trvncek, . tverk, L. Matteini, and M. Velli, Proton thermal energetics in the solar wind: Helios reloaded, JGR, VOL. 118, 13511365 (2013)

Hellinger et al.(2018). Hellinger, P., A. Verdini, S. Landi, L. Franci, and L. Matteini, VON KÁRMÁNHOWARTH EQUATION FOR HALL MAGNETOHYDRODYNAMICS: HYBRID SIMULATIONS, Astrophys. J. 857, L19 (2018)

Horbury et al.(2004). Horbury, T. S., Lucek, E. A., Balogh, A., Dandouras, I., and Rme, H., Motion and orientation of magnetic field dips and peaks in the terrestrial magnetosheath, J. Geophys. Res., 109, A09209 (2004) 
Howes et al.(2008). Howes, G. G. , S. C. Cowley, W. Dorland, G. W. Hammett, E. Quataert, and A. A. Schekochihin. A, model of turbulence in magnetized plasmas: Implications for the dissipation range in the solar wind, J. Geophys. Res. , 113(A12):A05103 (2008)

Howes et al.(2011). Howes, G. G., TenBarge, J. M., Dorland, W., Quataert, E., Schekochihin, A., Numata, R., \& Tatsuno, T. 2011, Phys. Rev. Lett., 107, 035004

Howes et al.(2014). Howes, G. G., K. G. Klein, and J. M. TenBarge, VALIDITY OF THE TAYLOR HYPOTHESIS FOR LINEAR KINETIC WAVES IN THE WEAKLY COLLISIONAL SOLAR WIND, ApJ, 789:106 (2014)

Howes et al.(2017). Howes, G. G., Klein, K. G. \& Li, T. C. Diagnosing collision-less energy transfer using field-particle correlations: Vlasov-Poisson plasmas. J. Plasma Phys. 83, 705830102 (2017)

Huang et al.(2010). Huang, S. Y., M. Zhou, F. Sahraoui, X. H. Deng, Y. Pang, Z. G. Yuan, Q. Wei, J. F. Wang, and X. M. Zhou, Wave properties in the magnetic reconnection diffusion region with high $\beta$ : Application of the k-filtering method to Cluster multispacecraft data, J. Geophys. Res., 115, A12211, doi:10.1029/2010JA015335 (2010)

Huang et al.(2014). Huang, S. Y., F. Sahraoui, X. H. Deng, J. S. He, Z. G. Yuan, M. Zhou, Y. Pang, \&, H. S. Fu, KINETIC TURBULENCE IN THE TERRESTRIAL MAGNETOSHEATH: CLUSTER OBSERVATIONS, The Astrophysical Journal Letters, 789:L28 (2014)

Huang et al.(2016). Huang, S. Y., F. Sahraoui, A. Retino, O. Le Contel, Z. G. Yuan, A. Chasapis, N. Aunai, H. Breuillard, X. H. Deng, M. Zhou, H. S. Fu, Y. Pang, D. D. Wang, R. B. Torbert, K. A. Goodrich, R. E. Ergun, Y. V. Khotyaintsev, P.-A. Lindqvist, C. T. Russell, R. J. Strangeway, W. Magnes, K. Bromund, H. Leinweber, F. Plaschke, B. J. Anderson, C. J. Pollock, B. L. Giles, T. E. Moore, and J. L. Burch, Geophys. Res. Lett., 43, 78507858 (2016)

Huang et al.(2017a). Huang, S.Y., Hadid, L.Z., Sahraoui, F., Yuan, Z. G., \& Deng, X. H., On the Existence of the Kolmogorov Inertial Range in the Terrestrial Magnetosheath Turbulence, The Astrophysical Journal Letters, 836:L10 (2017)

Huang et al.(2017b). Huang, S.Y., Sahraoui, F., Yuan, Z. G., et al., Magnetospheric Multiscale Observations of Electron Vortex Magnetic Hole in the Turbulent Magnetosheath Plasma, The Astrophysical Journal Letters, 836:L27 (2017)

Huang et al.(2018). Huang S. Y., Sahraoui, F., Yuan, Z. G., O. Le Contel, H. Breuillard, J. S. He , J. S. Zhao, H. S. Fu, M. Zhou, X. H. Deng, X. Y. Wang, J. W. Du, X. D. Yu, D. D. Wang, C. J. Pollock, R. B. Torbert, and J. L. Burch, Observations of Whistler Waves Correlated with Electron-scale Coherent Structures in the Magnetosheath Turbulent Plasma, ApJ, 861, 29 (2018)

Huang \& Sahraoui(2019). Huang S. Y. and F. Sahraoui, Testing the Taylor frozen-in-flow assumption at electron scales in solar wind turbulence, ApJ, 876 (2), 138 (2019)

Iroshnikov(1963). Iroshnikov, P. S., Astron. Zh. 40, 742 (1963)

Isenberg, Smith \& Matthaeus(2003). Isenberg, P.A., Smith, C.W., \& Matthaeus, W.H. 2003, Astrophys. J., 592, 564

Karimabadi et al.(2013). Karimabadi, H., V. Roytershteyn, M. Wan, W. H. Matthaeus, W. Daughton, P. Wu, M. Shay, B. Loring, J. Borovsky, E. Leonardis, S. C. Chapman, and T. K. M. Nakamura, Coherent structures, intermittent turbulence, and dissipation in high-temperature plasmas, Physics of Plasmas 20, 012303 (2013)

Karimabadi et al.(2014). Karimabadi, H., V. Roytershteyn, H. X. Vu, Y. A. Omelchenko, J. Scudder, W. Daughton, A. Dimmock, K. Nykyri, M. Wan, D. Sibeck, M. Tatineni, A. Majumdar, B. Loring, \& B. Geveci, The link between shocks, turbulence, and magnetic reconnection in collisionless plasmas, Phys. Plasmas, 21, 062308 (2014)

Kasper et al.(2008). , Kasper J. C., A. J. Lazarus, and S. P. Gary, Hot Solar-Wind Helium: Direct Evidence for Local Heating by Alfvn-Cyclotron Dissipation, Phys. Rev. Lett. 101, 261103 (2008)

Kiyani et al.(2009). Kiyani, K. H., Chapman, C., Khotyaintsev, Yu.V., Dunlop, M.W., \& Sahraoui, F. 2009, Phys. Rev. Lett., 103, 075006

Kiyani et al.(2013). Kiyani, K. H., Chapman, C., Sahraoui, F., B. Hnat, O. Fauvarque, and Yu. V. Khotyaintsev, ENHANCED MAGNETIC COMPRESSIBILITY AND ISOTROPIC SCALE INVARIANCE AT SUB-ION LARMOR SCALES IN SOLAR WIND TURBULENCE, The Astrophysical Journal, 763:10, 2013

Klein et al.(2017). Klein, K. G., Howes, G. G. \& Tenbarge, J. M. Diagnosing collisionless energy transfer using field-particle correlations: gyrokinetic turbulence. J. Plasma Phys. 83, 535830401 (2017)

Kobayashi et al.(2017). Kobayashi, S., F. Sahraoui, T. Passot, D. Laveder, P. L. Sulem, S. Y. Huang, P. Henri, and R. Smets, Three-dimensional Simulations and Spacecraft Observations of Sub-ion Scale Turbulence in the Solar Wind: Influence of Landau Damping, Astrophys. J., 839:122 (2017) 
Kolmogorov(1941). Kolmogorov, A. N. , Comptes Rendus (Doklady) de l'Academie des Sciences de l'URSS 30, 301 (1941)

Kraichnan(1965). Kraichnan, R. H. , Phys. Fluids 8, 13851387 (1965)

Kritsuk et al.(2013). Kritsuk, A. G., Wagner, R., \& Norman, M. L., J. Fluid Mech., 729, R1 (2013)

Kunz et al.(2014). Kunz, M. W., A. A. Schekochihin, and J. M. Stone, Firehose and Mirror Instabilities in a Collisionless Shearing Plasma, Phys. Rev. Lett. 112, 205003 (2014)

Lacombe et al.(2006). Lacombe, C., A. A. Samsonov, A. Mangeney, M. Maksimovic, N. CornilleauWehrlin, C. C. Harvey, J.-M. Bosqued, and P. Travnicek, Ann. Geophys. 24, 35233531 (2006)

Lacombe et al.(2014). Lacombe, L., O. Alexandrova, L. Matteini, O. Santolik, N. Cornilleau-Wehrlin, A Mangeney, Y. de Conchy, and M. Maksimovic, WHISTLER MODE WAVES AND THE ELECTRON HEAT FLUX IN THE SOLAR WIND: CLUSTER OBSERVATIONS, ApJ, 796:5 (2014)

Landau(1946). Landau, L. D., On the Vibrations of the Electronic Plasma. Journal of Physics , 10:25, 1946

Leamon et al.(1998). Leamon, R. J., Smith, C. W., Ness, N. F. , Matthaeus, W. H. \& Wong, H. K. 1998, J. Geophys. Res., 103, 4775

Liu et al.(2006). Liu, Y., J. D. Richardson, J. W. Belcher, and J. C. Kasper, Thermodynamic structure of collision-dominated expanding plasma: Heating of interplanetary coronal mass ejections, JGR, VOL. 111, A01102, doi:10.1029/2005JA011329 (2006)

Loureiro et al.(2013). Loureiro, N. F., A. A. Schekochihin, and A. Zocco, Fast Collisionless Reconnection and Electron Heating in Strongly Magnetized Plasmas, PRL 111, 025002 (2013)

MacBride, Smith \& Forman(2008). MacBride, B., Smith, C. W., \& Forman, M. 2008, Astrophys. J., 679, 1644

Mangeney et al.(2006). Mangeney, A, C Lacombe, M Maksimovic, A A Samsonov, N CornilleauWehrlin, C C Harvey, J-M Bosqued, and P Travnicek, Cluster observations in the magnetosheath - Part 1: Anisotropies of the wave vector distribution of the turbulence at electron scales, Ann. Geophys., 24:3507 (2006)

Marino et al.(2008). Marino, R., Sorriso-Valvo, L., Carbone, V. et al. 2008, Astrophys. J. Lett., 677, L71

Marino et al.(2011). Marino, R., Sorriso-Valvo, L., Carbone, V., et al. 2011, Planet. Space Sci., 59,592

Marsch(2006). Marsch, E. 2006, Living Rev. Sol. Phys., 3, 1

Matthaeus \& Goldstein(1982). Matthaeus W. H. \& Goldstein, M. L., 1982, J. Geophys. Res., 87:6011

Matthaeus et al.(1984). Matthaeus, W. H., J. J. Ambrosiano, M. L. Goldstein, Particle acceleration by turbulent magnetohydrodynamic reconnection, PRL, 53, 15 (1984)

Matthaeus et al.(1986). Matthaeus W. H., Goldstein, M.L., \& Lantz, S.R, Phys. Fluids 29, 1504 (1986)

Matthaeus et al.(1994). Matthaeus, W. H., S. Oughton, D. H. Pontius Jr., Y. Zhou, Evolution of energycontaining turbulent eddies in the solar wind, JGR 99, 19267 (1994)

Matthaeus et al.(2005). Matthaeus, W. H., S. Dasso, J. M. Weygand, L. J. Milano, C. W. Smith, and M. G. Kivelson, 2005, Phys. Rev. Lett., 95, 231101

Matthaeus et al.(2007). Matthaeus, W. H., B. Breech, \& P. Dmitruk, Astrophys. J. , 657 (2007)

Matthaeus \& Velli(2011). Matthaeus, W. H. and M. Velli, Who Needs Turbulence? A Review of Turbulence Effects in the Heliosphere and on the Fundamental Process of Reconnection, Space Sci. Rev., 160:145168 (2011)

Matthaeus et al.(2016). Matthaeus, W. H., J. M. Weygand, S. Dasso, Ensemble Space-Time Correlation of Plasma Turbulence in the Solar Wind, PRL 116, 245101 (2016)

Matteini et al.(2018). Matteini, L., , D. Stansby, T. S. Horbury, and C. H. K. Chen, On the 1/f Spectrum in the Solar Wind and Its Connection with Magnetic Compressibility, ApJL, 869:L32 (2018)

Meyrand \& Galtier(2010). Mayrand, R., \& Galtier, S. 2010, Astrophys. J.

Meyrand et al.(2019). Meyrand, M., A. Kanekar, W. Dorland, and A. A. Schekochihin, Fluidization of collisionless plasma turbulence, PNAS, 116 (4) 1185-1194 (2019)

Muller et al.(2013). Mller, D. R.G. Marsden, O.C. St. Cyr, H.R. Gilbert and the Solar Orbiter Team, Solar Orbiter Exploring the SunHeliosphere Connection, Solar Phys 285:2570, DOI 10.1007/s11207012-0085-7 (2013)

Narita et al.(2010a). Narita, Y., Glassmeier, K. H., Sahraoui, F., \& Goldstein, M. L. 2010a, Phys. Rev. Lett., 104, 171101

Narita et al.(2010b). Narita, Y., Sahraoui, F., Goldstein, M. L., \& Glassmeier, K. H. 2010b, J. Geophys. Res., 115, A04101, doi:10.1029/2009JA014742

Numata \& Loureiro(2015). Numata, R. and N. F. Loureiro, Ion and electron heating during magnetic reconnection in weakly collisional plasmas, J. Plasma Physics, vol. 81, 305810201 (2015) 
Osman et al.(2011). Osman, K. T., Wan, M., Matthaeus, W. H., J. M. Weygand, and S. Dasso, Anisotropic Third-Moment Estimates of the Energy Cascade in Solar Wind Turbulence Using Multispacecraft DataPhys. Rev. Lett., 107, 165001 (2011)

Osman et al.(2012). Osman, K. T., W. H. Matthaeus, B. Hnat, \& S. C. Chapman, Kinetic Signatures and Intermittent Turbulence in the Solar Wind Plasma, Phys. Rev. Lett., 108, 261103 (2012)

Osman et al.(2012). Osman, K. T., W. H. Matthaeus, M. Wan, and A. F. Rappazzo, Intermittency and Local Heating in the Solar Wind, Phys. Rev. Lett., 108, 261102 (2012)

Osman et al.(2013). Osman, K. T., W. H. Matthaeus, K. H. Kiyani, B. Hnat, and S. C. Chapman, Proton Kinetic Effects and Turbulent Energy Cascade Rate in the Solar Wind, Phys. Rev. Lett., 111, 201101 (2013)

Passot \& Sulem(2015). Passot, T., and P. L. Sulem, A MODEL FOR THE NON-UNIVERSAL POWER LAW OF THE SOLAR WIND SUB-ION-SCALE MAGNETIC SPECTRUM, ApJ, 812:L37 (2015)

Passot \& Sulem(2019). Passot, T., and P. L. Sulem, Imbalanced kinetic Alfvn wave turbulence: from weak turbulence theory to nonlinear diffusion models for the strong regime, Journal of Plasma Physics, Volume 85, Issue 3 (2019)

Perri et al.(2012). Perri, S., M. L. Goldstein, J. C. Dorelli, and F. Sahraoui, Detection of Small-Scale Structures in the Dissipation Regime of Solar-Wind Turbulence, PRL 109, 191101 (2012)

Perri et al.(2014). Perri, S., S. Servidio, A. Vaivads, F. Valentini, Numerical Study on the Validity of the Taylor Hypothesis in Space Plasmas, ApJ Supplement Series, Volume 231, Issue 1 (2017)

Pokhotelov et al.(2004). Pokhotelov, O. A., O. G. Onishchenko, R. Z. Sagdeev, M. A. Balikhin, and L. Stenflo, Parametric interaction of kinetic Alfven waves with convective cells, J. Geophys. Res. 109, A3 (2004)

Podesta et al.(2010). Podesta, J. J., J. E. Borovsky,, and S. P. Gary, 2010, A kinetic Alfven wave cascade subject to collisionless damping cannot reach electron scales in the solar wind at $1 \mathrm{AU}, \mathrm{ApJ}, 712$, $685(2010)$

Podesta et al.(2012a). Podesta, J. J. and J. M. TenBarge, Scale dependence of the variance anisotropy near the proton gyroradius scale: Additional evidence for kinetic Alfvn waves in the solar wind at 1 AUJ. Geophys. Res. 117, A10106 (2012)

Podesta et al.(2012b). Podesta, J. J., The need to consider ion Bernstein waves as a dissipation channel of solar wind turbulence, JGR, VOL. 117, A07101, doi:10.1029/2012JA017770 (2012)

Podesta(2013). Podesta, J. J., Evidence of Kinetic Alfvn Waves in the Solar Wind at 1 AU, Solar Phys, 286:529548, DOI 10.1007/s11207-013-0258-z (2013)

Politano \& Pouquet(1998). Politano, H., \& Pouquet, A. 1998, Phys. Rev. E, 57, 21

Pollock et al.(2016). Pollock, C., et al., Fast plasma investigation for magnetospheric multiscale, Space Sci. Rev., 199, 331, doi:10.1007/s11214-016-0245-4 (2016)

Retino et al.(2007). Retino, A., et al., Nature Phys., 3, 236238 (2007)

Richardson et al.(1995). Richardson, J.D., Paularena, I. K., Lazarus, A.J., \& Belcher, J. W., Evidence for a solar wind slowdown in the outer heliosphere?, Geophys. Res. Lett., 22, NO. 12, 1469-147 (1995)

Roberts et al.(2017). Roberts, O. W., Y. Narita, C. P. Escoubet, Direct Measurement of Anisotropic and Asymmetric Wave Vector Spectrum in Ion-scale Solar Wind Turbulence, ApJL, Volume 851, Issue 1 (2017)

Roytershteyn et al.(2015). Roytershteyn, V., H. Karimabadi, and A. Roberts, Generation of magnetic holes in fully kinetic simulations of collisionless turbulence, Phil. Trans. R. Soc. A, 373(20), 140151, doi:10.1098/rsta.2014.0151 (2015)

Russell (1972). Russell, C.T., 1972, Comments on the Measurement of Power Spectra of the Interplanetary Magnetic Field, in Solar Wind, Proceedings of a conference held March 21 26, 1971, at the Asilomar Conference Grounds, Pacific Grove, Calif., (Eds.) Sonett, C.P., Coleman, P.J., Wilcox, J.M., pp. 365374, NASA, Washington, DC

Sahraoui et al.(2003). Sahraoui, F. et al., 2003a, J. Geophys. Res.,108, 1335

Sahraoui et al.(2004). Sahraoui, F. et al., 2004, Ann. Geophys. 22, 2283

Sahraoui et al.(2006). Sahraoui, F., Belmont,G., Rezeau, L., Cornilleau-Wehrlin, N., Pinçon, J. L. , \& Balogh, A. 2006, Phys. Rev. Lett., 96, 075002

Sahraoui et al.(2007). Sahraoui, F., Galtier, S., Belmont,G. 2007, J. Plasma Phys., 73, 723

Sahraoui et al.(2008). Sahraoui, F., Diagnosis of magnetic structures and intermittency in space-plasma turbulence using the technique of surrogate data, Phys. Rev. E, 78, 026402 (2008)

Sahraoui et al.(2009). Sahraoui, F., Goldstein, M. L. , Robert, P. , Khotyaintsev, Y. V. 2009, Phys. Rev. Lett., 102, 231102 
Sahraoui et al.(2010a). Sahraoui, F., Goldstein, M. L., Belmont, G., Canu, P., \& Rezeau, L. 2010a, Phys. Rev. Lett., 105, 131101

Sahraoui et al.(2010b). Sahraoui, F., Belmont, G., Goldstein, M. L., \& Rezeau, L. 2010b, J. Geophys. Res., 115, A04206

Sahraoui et al.(2012). Sahraoui, F., Belmont, G., \& M. L. Goldstein, NEW INSIGHT INTO SHORTWAVELENGTH SOLAR WIND FLUCTUATIONS FROM VLASOV THEORY, The Astrophysical Journal, 748:100 (2012)

Sahraoui et al.(2013). Sahraoui, F., Huang, S., \& Goldstein, M. L., Rétino, A., Robert, P., and De Patoul, J., ApJ, 777:15, 2013

Sahraoui \& Huang(2019). Sahraoui, F., \& S. Y. Huang, On the broadening of the spectral slopes in subion scales turbulence in the solar wind, submitted (2019)

Salem et al.(2012). Salem, C. S., G. G. Howes, D.Sundkvist, S. D. Bale, C. C. Chaston, C. H. K. Chen, \& F. S. Mozer, Astrophys. J. Lett. 745, L9 (2012)

Schekochihin et al.(2009). Schekochihin, A., Cowley, S. C., Dorland, W., Hammett, G. W., Howes, G. G., Quataert, E., \& Tatsuno, T. 2009, Astrophys. J.s, 182, 310

Schekochihin et al.(2016). Schekochihin, A. A., J. T. Parker, E. G. Highcock, P. J. Dellar, W. Dorland and G. W. Hammett, Phase mixing versus nonlinear advection in drift-kinetic plasma turbulence, J. Plasma Phys., vol. 82, 905820212 (2016)

Servidio et al.(20111). Servidio, S., A. Greco, W. H. Matthaeus, K. T. Osman, and P. Dmitruk, Statistical association of discontinuities and reconnection in magnetohydrodynamic turbulence, JGR, OL. 116, A09102,doi:10.1029/2011JA016569 (2011)

Servidio et al.(2014). Servidio, S., K.T.Osman, F. Valentini, D. Perrone, F. Califano, S. Chapman, W. H. Matthaeus, \& P. Veltri, PROTON KINETIC EFFECTS IN VLASOV AND SOLAR WIND TURBULENCE, Astrophys. J. Lett., 781:L27 (2014)

Servidio et al.(2017). Servidio, S., A. Chasapis, W. H. Matthaeus, D. Perrone, F. Valentini, T. N. Parashar, P. Veltri, D. Gershman, C. T. Russell, B. Giles, S. A. Fuselier, T. D. Phan, and J. Burch, Magnetospheric Multiscale Observation of Plasma Velocity-Space Cascade: Hermite Representation and Theory, PRL 119, 205101 (2017)

Slavin \& Holzer(1981). SLAVIN, J. A., and R. E. HOLZER, Solar Wind Flow About the Terrestrial Planets: Modeling Bow Shock Position and Shape, JGR, VOL. 86, NO. A13, PAGES 11,401-11,418 (1981)

Smith et al.(2006). Smith, C.W., et al. 2006, Astrophys. J. Lett., 645, L85

Smith et al.(2009). Smith, C.W., et al. 2009, Phys. Rev. Lett., 103; 201101

Sorriso-Valvo et al.(2007). Sorriso-Valvo, L., Marino, R., Carbone, V., et al. 2007, Phys. Rev. Lett., 99, 115001

Stawarz et al.(2009). Stawarz, J. E., Smith, C. W., Vasquez, B. J., et al. 2009, Astrophys. J., 697, 1119

Stawicki et al.(2001). Stawicki, O., Gary, S. P., \& Li, H. 2001, J. Geophys. Res., 106, 8273

Stverak et al.(2015). tverk, , P. M. Trvncek, and P. Hellinger, Electron energetics in the expanding solar wind, J. Geophys. Res. Space Physics, 120, 81778193, doi:10.1002/2015JA021368 (2015)

Sulem \& Passot(2015). Sulem, P. L. \& Passot, T., Landau fluid closures with nonlinear large-scale finite Larmor radius corrections for collisionless plasmas, J. Plasma Physics, 81, (2015)

Sundvist et al.(2007). Sundkvist, D., A. Retino, A. Vaivads, \& S. Bale, Dissipation in Turbulent Plasma due to Reconnection in Thin Current Sheets, Phys. Rev. Lett. 99, 025004 (2007)

Tjulin et al.(2005). Tjulin, A., Pinçon, J.L., Sahraoui, F., André, M., \& Cornilleau-Wehrlin, N. 2005, J. Geophys. Res., 110, A11224, doi:10.1029/2005JA011125

Tenbarge et al. (2012). , TenBarge, J. M. , J. J. Podesta, K. G. Klein, \& G. G. Howes, INTERPRETING MAGNETIC VARIANCE ANISOTROPY MEASUREMENTS IN THE SOLAR WIND, ApJ, 753:107 (2012)

Tenbarge \& Howes (2013). TenBarge, J. M, \& G. G. Howes, CURRENT SHEETS AND COLLISIONLESS DAMPING IN KINETIC PLASMA TURBULENCE, ApJL 771:L27 (2013)

Vaivads et al.(2016). Vavaids, A., et al., Turbulence Heating ObserveR: satellite mission proposal, J. Plasmas Phys. 82, 905820501 (2016)

Verscharen et al.(2019). Verscharen, D., R. T. Wicks, et al., A case for Electron Astrophysics, arXiv:1908.02206 (2019)

Verdini\& Grappin(2016). Verdini, A. and R. Grappin, BEYOND THE MALTESE CROSS: GEOMETRY OF TURBULENCE BETWEEN 0.2 AND 1 AU, Astrophysical Journal, 831:179 (2016)

Voitenko \& De Keyser(2016). Voitenko, Y., and J. De Keyser, MHDKINETIC TRANSITION IN IMBALANCED ALFVNIC TURBULENCE, ApJL, 832:L20 (2016) 
Wan et al.(2010). Wan, M., S. Servidio, S. Oughton, and W. H. Matthaeus, The third-order law for magnetohydrodynamic turbulence with shear: Numerical investigation, PHYSICS OF PLASMAS 17, 052307 (2010)

Wicks et al.(2013). Wicks, R. T., A. Mallet, T. S. Horbury, C. H. K. Chen, A. A. Schekochihin, and J. J. Mitchell, Alignment and Scaling of Large-Scale Fluctuations in the Solar Wind, PRL 110, 025003 (2013)

Velli et al.(1989). Velli, M., R. Grappin, \& A. Manegeney, Phys. Rev. Lett., 1807 (1989)

Verdini et al.(2012). Verdini, A., Grappin, G., Pinto, R., \& Vellim, M., The Astrophysical Journal 33, 750 (2012)

Yao et al.(2017). Yao, S. T., X. G. Wang, Q. Q. Shi, T. Pitknen, M. Hamrin, Z. H. Yao, Z. Y. Li, X. F. Ji, A. De Spiegeleer, Y. C. Xiao, A. M. Tian, Z. Y. Pu, Q. G. Zong, C. J. Xiao, S. Y. Fu, H. Zhang, C. T. Russell, B. L. Giles, R. L. Guo, W. J. Sun, W. Y. Li, X. Z. Zhou, S. Y. Huang, J. Vaverka, M. Nowada, S. C. Bai, M. M. Wang, J. Liu, Observations of kinetic-size magnetic holes in the magnetosheath, J. Geophys. Res. Space Physics, 122, 19902000, doi:10.1002/2016JA023858 (2017)

Yao et al.(2019). Yao, S. T., Q. Q. Shi, Z. H. Yao, J. X. Li, C. Yue, X. Tao, A. W. Degeling, Q. G. Zong, X. G. Wang, A. M. Tian, C. T. Russell, X. Z. Zhou, R. L. Guo, I. J. Rae, H. S. Fu, H. Zhang, L. Li, O. Le Contel, R. B. Torbert, R. E. Ergun, P.A. Lindqvist, C. J. Pollock and B. L. Giles, Waves in KineticScale Magnetic Dips: MMS Observations in the Magnetosheath, Geophysical Research Letters, 46, 2, 523-533 (2019)

Yordanova et al.(2008). Yordanova, E., A. Vaivads, M. André, S. C. Buchert, \& Z. V., Magnetosheath Plasma Turbulence and Its Spatiotemporal Evolution as Observed by the Cluster Spacecraft, Phys. Rev. Lett., 100, 205003 (2008) 